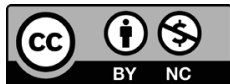

Licenciado sob uma licença Creative Commons

ISSN 2175-6058

DOI: https://doi.org/10.18759/rdgf.v21i3.1825

\title{
EMERGENZE E ORDINAMENTO COSTITUZIONALE ITALIANO
}

EMERGENCIES AND THE ITALIAN CONSTITUTIONAL ORDER

\section{EMERGÊNCIAS E A ORDEM CONSTITUCIONAL ITALIANA}

\author{
Ugo Adamo \\ Silvio Gambino
}

\section{SOMMARIO}

Il contributo propone un'analisi critica dei riflessi che si possono determinare nell'ordinamento costituzionale per il verificarsi di situazioni emergenziali che richiedono l'adozione di provvedimenti idonei a farvi fronte. In tale prospettiva, lo studio prende in considerazione le emergenze terroristiche e quelle sanitarie per valutarne differenze e analogie, stanti le innumerevoli conseguenze che le decisioni adottate in tali contesti producono sul bilanciamento tra libertà individuali e interessi della collettività, sul sistema delle fonti e sulla stessa forma di Stato e di governo. La tenuta costituzionale regge quando il sistema è solido e quando la democrazia è matura, essendo possibile limitare i diritti nel bilanciamento con altri diritti, per come viene riconosciuto dallo stesso ordinamento convenzionale; ciò è più che evidente dinanzi agli sviluppi ordinamentali verificatisi in alcuni Paesi europei, come la Polonia e l'Ungheria, che strumentalizzano la crisi pandemica per mettere in discussione la rule of law.

Parole chiave: Libertà individuale. Interessi collettivi. Pandemia. 


\section{ABSTRACT}

The contribution proposes a critical analysis of the consequences that can be determined in the constitutional legal system upon the occurrence of emergencies that require the adoption of suitable measures to deal with them. In this perspective, the study takes into consideration terrorist and health emergencies. It evaluates the differences and similarities in these contexts, due to the innumerable consequences that the decisions adopted produce in balancing between individual freedoms and collective interests, on the system of sources, and the form of state and government. The constitutional system holds up if it is concrete and if democracy is mature. Upon the occurrence of these conditions, it is possible to limit the rights in the balance with other rights, respecting what is recognized by the same constitutive system. This can be seen in the legal developments that occurred in some European countries, such as Poland and Hungary, which exploit the pandemic crisis to question the rule of law.

Keywords: Individual freedom. Collective interests. Pandemic.

\section{RESUMO}

A contribuição propõe uma análise crítica das consequências que podem ser determinadas no ordenamento jurídico constitucional sobre a ocorrência de emergências que requeiram a adoção de medidas cabíveis para enfrentá-las. Nessa perspectiva, o estudo leva em consideração as emergências terroristas e de saúde. Assim, avalia as diferenças e semelhanças nestes contextos, devido às inúmeras consequências que as decisões adotadas produzem no equilíbrio entre as liberdades individuais e os interesses coletivos, sobre o sistema de fontes, e a forma de Estado e de governo. 0 sistema constitucional se mantém se for concreto e se a democracia for madura. Com a ocorrência dessas condições, é possível limitar os direitos em equilíbrio com os demais direitos, respeitando-se o que é reconhecido pelo mesmo sistema constitutivo. Isso pode ser visto nos desenvolvimentos jurídicos ocorridos em alguns países europeus, como a Polônia e a Hungria, que exploram a crise pandêmica para questionar o Estado de Direito.

Palavras-chaves: Liberdade individual. Interesses coletivos. Pandemia. 


\section{SU ALCUNE PROBLEMATICHE COSTITUZIONALI ORIGINATE DALLA 'LEGISLAZIONE DI EMERGENZA ANTITERRORISMO' E ORA DALLA 'LEGISLAZIONE DI EMERGENZA EPIDEMIOLOGICA'. ALCUNE PREMESSE}

Il nostro intento è quello di offrire una analisi della possibile dinamica dei rapporti fra libertà e sicurezza ${ }^{1}$, muovendo in particolare dalle problematiche sollevate dal contrasto della pandemia da CoViD-19 in corso (e in special misura avendo a riguardo il caso italiano) a confronto con quelle del contrasto del terrorismo.

La (stretta) connessione fra libertà personali e sicurezza suggerisce di iniziare la riflessione con il richiamo di due sentenze nelle quali è ben argomentato il vincolo del principio di legalità nella conformazione dello Stato democratico e di diritto. Nella prima delle due decisioni (Trabelsi c. Italia, 2010), la Corte europea dei diritti dell'uomo sentenzia che «la forza della democrazia si misura proprio quando, pur in presenza di un pericolo incombente, si continua ad operare nella legalità». Tale indirizzo viene confermato dalla medesima Corte con riguardo al divieto di espulsione, allontanamento o estradizione di imputati o di condannati verso Paesi nei quali sia prevista la pena di morte e venga utilizzata la tortura o i trattamenti inumani e degradanti; un divieto che è da assumere come assoluto anche a fronte di emergenze terroristiche o di altra natura (Saadi c. Italia, 2008). A tale indirizzo si conforma la Corte di Cassazione (SS. UU. civ., 2009) quando fa proprio l'indirizzo per il quale i

«diritti umani fondamentali non possono essere oggetto di ponderazione valutativa con nessun altro interesse pubblico, fosse anche quello della sicurezza degli Stati nazionali, cosicché nessun margine di discrezionalità spetta alle pubbliche amministrazioni, che devono valutare l'istanza di asilo da parte dello straniero».

Con riguardo specifico alla legislazione intervenuta sul tema della presente analisi, si osserva come, da più di un trentennio, - al di qua e al di là dell'Oceano - sono state previste misure securitarie (preventive e repressive) del fenomeno criminale terroristico nella totalità dei Paesi di democrazia pluralista. Rispetto a tali misure e al rischio (a esse) 
immanente di possibili antinomie fra (esigenze della) sicurezza e (cultura della) legalità, la dottrina costituzionale si era da tempo interrogata sulla stessa necessarietà e sulla relativa conformità costituzionale di una «Costituzione di emergenza ${ }^{2}$, che predisponesse princìpi e regole idonee a «salvaguardare libertà e diritti civili di fronte al pericolo del terrorismo», assicurando agli Esecutivi dei singoli Paesi di esercitare la garanzia della sicurezza senza apportare danni duraturi ai diritti individuali, e comunque senza varcare, in tale contrasto del terrorismo, i limiti imposti dalla «decenza». In un simile scenario, complesso e aperto all'apporto del legislatore e del giudice costituzionale, l'interrogativo ricorrente che l'opinione pubblica e la dottrina costituzionale si era posto, a partire dalla legislazione di contrasto del terrorismo (legge Reale, in primis), era quello di come rispondere alle gravi incertezze in cui veniva a trovarsi il legislatore quando era chiamato a rispondere al problema, ma anche alle ansie sociali con cui lo stesso si presentava (e si rappresentava): 'Meno libertà, più sicurezza'?

Tale interrogativo si fa ora più assillante rispetto a quanto non accadesse per le misure di contrasto del fenomeno terroristico, dovendo ora le misure governative contrastare la diffusione di un virus che aggredisce in modo letale la salute pubblica, assumendo le forme del distanziamento sociale e della compressione di diverse libertà fondamentali (fra cui circolazione, associazione, riunione, culto, iniziativa economica, istruzione). Che poi tali misure emergenziali di contrasto epidemiologico assumano (anche) la forma del decreto del Presidente del Consiglio dei Ministri (d'ora in avanti dPCM) e non piuttosto (solo) quella - corretta costituzionalmente - del decreto-legge (d'ora in avanti d.-l.), nel merito, costituisce un problema di ordine costituzionale supplementare rispetto a quelli già evocati per la legislazione di contrasto del terrorismo nella quale a rilevare in modo problematico era soprattutto la violazione dei limiti costituzionali (della riserva di legge e della riserva di giurisdizione) fissati dalle previsioni dell'art. 13 della Costituzione. Un simile (ancorché essenziale) accenno al sistema costituzionale delle fonti e al principio di legalità è apparso necessario al momento di riflettere sui limiti all'esercizio del potere normativo da parte dei soggetti titolari del potere di decidere nelle emergenze, in quanto, in assenza di previsioni costituzionali in tema 
di 'stato d'eccezione', queste ultime rilevano al diritto costituzionale interno come 'meri' 'casi straordinari di necessità e di urgenza', legittimando per la relativa disciplina il ricorso da parte del Governo all'art. 77, c. 2, Cost. Per risultare rispettosi della natura fondamentale delle previsioni costituzionali di tutela dei diritti fondamentali, tali limiti impongono al legislatore (e imporranno al Giudice delle leggi) di adottare scelte che si fondino sul bilanciamento fra i diversi beni oggetto della protezione costituzionale quando gli stessi vengano in rilievo nella concretezza della disciplina legislativa (è il caso appunto della libertà personale e del suo potenziale contrasto con l'ordine pubblico e la moralità pubblica, ovvero il caso della emergenza epidemiologica in corso, nella quale a rilevare è il contrasto temporaneo, potenziale o concreto, fra il bene salute (privata e pubblica) e la compressione della libertà di circolazione e delle altre manifestazioni della libertà costituzionalmente positivizzate.

In un simile quadro di bilanciamenti nessuno di tali beni costituzionali può assumere pretese 'tiranniche' nei confronti degli altri, imponendosi, in tal modo, una lettura valoriale e giuspositiva dei principi e dei diritti fondamentali che li coglie non in modo assolutistico ma nell'ottica (più 'mite') dei «diritti fra i diritti» ${ }^{3}$. Una esemplificazione di tali bilanciamenti risulta osservabile nella giurisprudenza costituzionale in tema di diritti sociali, ove il parametro di riferimento comparativo per la Corte è costituito dal rispetto del loro 'nucleo essenziale' secondo il fondamentale parametro della dignità umana. Recenti indirizzi giurisprudenziali relativi a diritti costituzionali che la Corte ha ora qualificato come 'incomprimibili', tuttavia, hanno operato una rilevante svolta argomentativa destinata a superare molte delle criticità e dei dubbi che erano stati fin qui sollevati in tema di bilanciamento fra beni costituzionali come quelli costituiti dalla finanza pubblica e dalla garanzia dei diritti fondamentali sociali ${ }^{4}$.

I limiti richiamati nell'esercizio del potere decisionale nel quadro delle crisi emergenziali costituiscono un argine rigido alla separazione dei poteri (e in particolare del potere legislativo e di quello regolamentare e amministrativo) ponendosi come parametro di riferimento per il Giudice costituzionale (e prima ancora per lo stesso Esecutivo). L'emergenza epidemiologica non ha avuto ancora il tempo necessario per stimolare una giurisprudenza (significativa e consolidata) nei più diversi ambiti 
dell'azione pubblica (amministrativa, penale, costituzionale), ma non vi è dubbio che la stessa non potrà che intervenire nel prosieguo della disciplina di contrasto dell'emergenza sanitaria.

Nel bilanciare le libertà personali degli indagati e dei sospetti di terrorismo e le esigenze di sicurezza giuridica dell'intero corpo sociale come si potrà ricordare - il Giudice delle leggi era pervenuto a sancirne la legittimità costituzionale nel quadro di una valorizzata 'proporzionalità' e 'temporaneità' delle misure di limitazione delle libertà per come sancite nella legislazione (speciale) di contrasto del terrorismo; omologamente potrà ora fare con riguardo alle variegate limitazioni della libertà sancite nella normativa di contrasto dell'emergenza sanitaria. Interrogativi simili, al momento di tipo dottrinario, sono ora posti con riguardo alla normativa di contrasto dell'emergenza epidemiologica (dd.-ll., ddPCM, ordinanze di protezione civile, dei presidenti delle giunte regionali e dei sindaci). Tale normativa, susseguitasi in un breve lasso temporale (e in modo spesso discutibile con riguardo alla tipologia delle fonti utilizzata e con riguardo alla stessa sovrapposizione fra fonti regionali e fonti statali), disciplina, fra l'altro, misure di confinamento domestico nonché modalità tecnologiche per il controllo da remoto del rispetto della normativa sul distanziamento sociale (e di tutte le altre misure per il contenimento della epidemiologia), le une e le altre ritenute necessarie per contrastare la diffusione della pandemia.

Le misure adottate in un simile quadro sono state tali da ingenerare un forte dibattito fra Stato e regioni sul concreto riparto delle competenze in materia sanitaria con riguardo alla disciplina di contrasto della pandemia, accompagnato da un (parimenti intenso) dibattito relativamente alla fonte utilizzata da parte del Presidente del Consiglio per disporre le misure ritenute necessarie (sulla base delle valutazioni dell'Istituto Superiore di Sanità, e non solo). Riguardando (tali misure) lo stesso livello costituzionale dei diritti fondamentali, si è fatto opportunamente osservare da parte di una dottrina costituzionale ampiamente concorde sul punto che lo strumento per contrastare l'emergenza sanitaria, per le ragioni richiamate, non poteva essere certo quello amministrativo (dPCM), inidoneo per la sua derivazione monocratica dal Presidente del Consiglio a riconoscere le ragioni del controllo necessario sul medesimo 
(da parte del Parlamento e del Presidente della Repubblica), apparendo giustificata l'esigenza, nel quadro di una forma di Stato parlamentare, che l'assunzione di decisioni in materia di libertà fondamentali debba individuare la sua sede più appropriata nella legge ${ }^{5}$.

\section{SICUREZZA SENZA LIBERTÀ. LA 'COSTITUZIONE PER L'EMERGENZA' COME (POSSIBILE) RIMEDIO (DE JURE CONDENDO) ALLE DERIVE LIBERTICIDE DELLE STRATEGIE DI LOTTA AL TERRORISMO E ALLE PANDEMIE?}

Prima di volgere lo sguardo alla legislazione italiana emergenziale nelle due principali stagioni (di contrasto del terrorismo e della emergenza epidemiologica), è opportuno richiamare la tematica fondamentale posta, nel dibattito costituzionale, dalle relazioni (talora incerte) fra le finalità oggetto di protezione nello Stato costituzionale e i limiti sanciti costituzionalmente al sistema delle fonti (riserva di legge, riserva di giurisdizione) - limiti che si riflettono appunto sulla disciplina del sistema delle fonti e sulla costituzionalizzazione di limiti puntuali alla funzione normativa dell'Esecutivo (e naturalmente dello stesso Legislativo) nella disciplina dei diritti e delle libertà fondamentali -. L'insieme di tali relazioni merita di essere ora richiamato con riguardo al bilanciamento fra garanzie costituzionali delle manifestazioni della libertà personale e bene costituzionale della «sicurezza» ${ }^{6}$. In tema, in dottrina è in corso da più tempo un dibattito circa la qualificazione della sicurezza nella cornice costituzionale volto a dare una risposta appagante all'interrogativo di chi si chiede se «il soddisfacimento del bene 'sicurezza' inteso in senso soggettivo può comprimere la tutela dei beni specifici costituzionalmente protetti, subordinando il bilanciamento tra i medesimi all'obiettivo di rafforzare la percezione soggettiva della sicurezza stessa» ${ }^{7}$.

La riflessione muove da una prima prospettiva secondo la quale la sicurezza deve essere intesa come «valore supremo», «super-principio» dell'ordinamento costituzionale, «sostrato di fondo immanente alle stesse garanzie costituzionali dei diritti» ${ }^{8}$. Tale lettura porta ad assegnare alla 
sicurezza «una sostanza diversa rispetto ai diritti costituzionali che ne impedisce il conflitto ma, soprattutto, che pone la sicurezza al di fuori della possibilità di un bilanciamento ${ }^{9}$, in quanto non può aversi «una sicurezza attenuata, poiché la sicurezza bilanciata è una non sicurezza» ${ }^{10}$. Diversamente da tale prima prospettiva, si muovono altre prospettive ${ }^{11}$ nelle quali si perviene alla valorizzazione della dimensione individuale/ personale della sicurezza e in tale ottica alla qualificazione della sicurezza come situazione giuridica soggettiva perfetta, come diritto soggettivo pieno.

Pur considerando legittime le esigenze poste dalla percezione della sicurezza pubblica, tuttavia, tale lettura registra forti resistenze, portando a sottolineare problematicamente come sia 'inesatto e addirittura fuorviante' parlare di un diritto soggettivo alla sicurezza. Chiarificatore nell'ottica di tale indirizzo (che nega ogni riferibilità della sicurezza al parametro dei diritti inviolabili) appare l'iter argomentativo del Giudice delle leggi italiano quando osserva che «spetta esclusivamente alla discrezionalità del legislatore determinare, nel rispetto del principio della riserva di legge stabilito dall'art. 13, comma 2, Cost., i casi in cui il giudice può disporre restrizioni della libertà personale, ed è pure riservata alla discrezionalità del legislatore la determinazione dei casi eccezionali di necessità e urgenza in cui possono essere adottati provvedimenti provvisori limitativi della libertà personale ai sensi dell'art. 13, comma 3, Cost.» (Corte cost., ord. cost. n. 187/2001). Da ciò consegue che, pur potendosi parlare di una «funzione di sicurezza», non dovrebbe ancora potersi parlare di «diritto alla sicurezza», non potendo la sicurezza pubblica «costituire il contenuto di un diritto della persona o di un diritto sociale ... (costituendo la stessa null'altro che) ... uno stato psicologico collettivo da cui promana un interesse diffuso, la cui tutela è di competenza esclusiva dello Stato» ${ }^{12}$.

In tema, si fa parimenti osservare come sia necessario valorizzare le esigenze (teoriche e pratiche) poste dalla necessaria «diffidenza nei confronti dell'ossessione securitaria» ${ }^{13}$ cogliendo, al contempo, le conseguenze dinamiche dell'evoluzione della forma statuale rispetto alle garanzie assicurate dai principi liberal-democratici del diritto alla sicurezza nelle sue diverse declinazioni costituzionali. Tali modulazioni riguardano il bene della sicurezza nei suoi variegati profili: in senso stretto, come «ordine pubblico e sicurezza»e, in una concezione più 
ampia e dinamica (funzionale alla stessa protezione dei diritti sociali), come interesse della collettività, idonea a bilanciare i diritti di libertà in funzione della garanzia dell'ordinato vivere civile, per come argomenta la Corte costituzionale.

Nella presente fase evolutiva dello Stato sociale, la sicurezza, intesa come situazione giuridica piena, potrà essere meglio intesa se viene colta in modo meno distante dalle esigenze poste dalla tutela del bene della/e libertà personale/i e se è inquadrata alla luce di una convincente lettura (incentrata anche sulla ricerca dell' «altro volto della sicurezza» ${ }^{14}$ ) che la colga come «certezza del diritto», come «sicurezza nel diritto»15, superando in tal modo quell'approccio statico ('sicurezza e ordine pubblico') che era insito nella lettura risalente di tale bene giuridico, a favore di una valorizzazione del 'presupposto promozionale' del concetto di sicurezza come pretesa costituzionalmente garantita dei soggetti verso i pubblici poteri, volta a conseguire la rimozione degli ostacoli ${ }^{16}$ che si frappongano all'effettivo godimento dei diritti, e con essa la realizzazione della «libertà dal bisogno» ${ }^{17}$.

Nel quadro delle risposte che la dottrina ha offerto rispetto alle torsioni ordinamentali provocate dalle emergenze (dapprima quella del terrorismo e ora quella provocata dalla pandemia e dalla gravi crisi economica che l'accompagna), la sicurezza, in accordo con taluni orientamenti volti alla previsione di una deroga costituzionale (temporanea) a fronte (- e per superarle -) delle situazioni di emergenza (terroristica e ora epidemiologica), sembrerebbe in tal modo poter comunque assurgere - se non anche al rango di diritto fondamentale - almeno a quello di 'nuovo' valore costituzionale ${ }^{18}$.

Rispetto a (possibili) reazioni scomposte della popolazione riguardata da attacchi terroristici e ora dagli effetti traumatici delle crisi economiche prodotte dalle pandemie (tali da indurre anche il rischio di rottura dell'ordine pubblico e della stessa democrazia), una parte della dottrina suggerisce di positivizzare nel testo costituzionale una clausola volta a disciplinare gli 'stati di emergenza', mediante la previsione di una 'Costituzione per l'emergenza' «che permetta di adottare misure operative efficaci per prevenire un secondo attentato nel breve termine ma che, con fermezza, stabilisca un limite alla loro durata» ${ }^{19}$ - con riguardo alla 
ipotesi di emergenza dovuta al terrorismo -, ovvero che consenta di adottare misure di contenimento della diffusione della pandemia senza mettere in questione l'ordinato esercizio del potere normativo nei casi di necessità e urgenza, rispettivamente, da parte del Parlamento e del Governo. Non in modo casuale, in tale ottica, l'analisi dello studioso nordamericano ove si esplicitava un simile orientamento era intitolato Prima del prossimo attacco, preoccupandosi - l'Autore - di specificare come, oltre a complessi (e garantistici) limiti procedurali previsti nella revisione del testo costituzionale, la clausola di emergenza dovesse preoccuparsi di disciplinare la natura temporanea dello "stato di emergenza» ${ }^{20}$, evitando in tal modo di autorizzare limitazioni durature dei diritti. Per come si argomenterà - però, e da qui in avanti - la costituzionalizzazione dello stato di emergenza (che rimane pur sempre tra le opzioni politiche e di politica del diritto, stante la consapevolezza che tale scelta fu esplicitamente messa da parte nel corso del dibattito in sede costituente) non risulta doverosa $^{21}$, in quanto la Costituzione (e la giurisprudenza ordinaria e costituzionale degli ultimi decenni) ha dimostrato come essa stessa non perda (non abbia perso) il carattere prescrittivo neanche in una situazione di fortissimo stress.

\section{L'ESPERIENZA ITALIANA IN TEMA DI LEGISLAZIONE DI CONTRASTO AL COVID-19}

Ogni emergenza ha una sua specificità e può sottoporre a variazioni diverse le tensioni che si possono generare sulla tenuta complessiva della Carta costituzionale, dal momento che non si può immaginare di poter dare una risposta eguale e generale valida per ogni situazione per il solo fatto che venga definita di emergenza nazionale.

In particolare, l'emergenza epidemica ha il carattere della mutevolezza, vale a dire che le misure normative da adottare al fine della tutela della salute - che come vedremo è il diritto fondamentale intorno al quale si definisce il punto del bilanciamento - devono accompagnare l'attività pandemica e non possono essere prefissate aprioristicamente (una volta per tutte) per l'intera fase emergenziale: il diacronico è embricato nel 
sincronico. L'epidemiologia (nel caso italiano) ci insegna che una fase di latenza è seguita da una di picco del contagio, quindi da una situazione di plateau e poi da una fase discendente ma non fino alla scomparsa del virus (e delle sue potenzialità di contagio), la cui costante latenza può generare a sua volta un moto ricorsivo, almeno fino a quando non sia messa a punto una cura specifica o meglio ancora un vaccino.

La risposta normativa all'emergenza epidemiologica deve essere inquadrata in una cornice di assoluta novità e di mancanza di strumenti già disponibili per farvi fronte, benché già dal 2003 avrebbero dovuto essere compiutamente definiti dei piani anti-pandemici. Inoltre, il dato normativo registra un grado di flessibilità dovuto al fatto che si è dinanzi ad un processo (nazionale) ${ }^{22}$ e non ad un fatto verificatosi in modo temporalmente puntuale ${ }^{23}$ e spazialmente omogeneo. L'analisi delle misure normative adottate, quindi, deve tener conto di una iniziale scarsa conoscenza del fenomeno solo progressivamente ampliatasi, ma questa circostanza fattuale non per ciò solo può affievolire la prescrittività che è del testo costituzionale, che sta dimostrando, ancora una volta, di sapere «offrire alle Istituzioni e ai cittadini la bussola necessaria a navigare 'per l'alto mare aperto' dell'emergenza e del dopo-emergenza» 24 .

Questa premessa ci permette almeno altre due considerazioni. In primo luogo (nel momento in cui si scrive si è nominalmente nella c.d. 'fase 2 ' ${ }^{25}$, non ci si trova in nessuno stato d'eccezione, ma di necessità; in secondo luogo, e per il motivo appena detto, la nostra analisi dà per assodato come l'attuale fase sia eccezionale e quindi le misure adottate per farvi fronte debbano essere per definitionem temporalmente circoscritte e per nessun motivo possano rientrare in quella che sovente viene definita emergenza perenne: situazione questa che sempre più appare connaturata alla fluidità del mondo contemporaneo. È certo che una situazione d'emergenza che non sia circoscritta in un tempo definito risulti per ciò solo come una situazione incapace di favorire una ricerca di ragionevolezza delle misure (eccezionali) limitatrici delle libertà costituzionali, oltre a non poter più essere definita d'emergenza (si pensi a situazioni che seppure divenute di fatto 'ordinarie' continuano a essere definite 'emergenziali': rifiuti, sanità, crisi economiche, lotta al terrorismo $)^{26}$. 
Inoltre, le conclusioni a cui si giungerà in ordine a una complessiva legittimazione delle misure limitatrici delle libertà costituzionali non potranno essere assunte come un 'precedente' a cui riferirsi al fine di rispondere a esigenze di necessità di altro ordine (pubblico, sociale, economico, politico $)^{27}$.

Per iniziare la disamina, i primi atti da cui partire sono quello della Organizzazione Mondiale della Sanità che ha dichiarato lo stato di emergenza di sanità pubblica di rilievo internazionale il 30 gennaio $2020^{28}$, e quello (seguente e conseguente) della Dichiarazione dello stato di emergenza ${ }^{29}$ in conseguenza del rischio sanitario connesso all'insorgenza di patologie derivanti da agenti virali trasmissibili, deliberato dal Consiglio dei Ministri (datato il giorno successivo e che resterà in vigore, al momento stanti le due proroghe, fino al prossimo 31 gennaio) secondo quanto disposto dall'art. 7, c. 1, lett. c), d.lgs. n. 1 del 2 gennaio 2018 Codice della Protezione civile $e^{30}$, e, quindi, dall'art. 25 per cui le ordinanze di protezione civile - che non sono soggette ad alcun controllo preventivo di legittimità - possono adottarsi

«in deroga ad ogni disposizione vigente, nei limiti e con le modalità indicati nella deliberazione dello stato di emergenza e nel rispetto dei principi generali dell'ordinamento giuridico e delle norme dell'Unione europea. Le ordinanze sono emanate acquisita l'intesa delle Regioni e Province autonome territorialmente interessate e, ove rechino deroghe alle leggi vigenti, devono contenere l'indicazione delle principali norme a cui si intende derogare e devono essere specificamente motivate».

Fatta tale precisazione e prescindendo dall'articolato appena richiamato, a fronte dell'emergenza (anche) sanitaria si è proceduto con atti primari ${ }^{31}$ sui quali si fonda la quasi totalità della normativa emergenziale caratterizzata da quello che definiremo il tandem decretolegge/decreto Presidente del Consiglio dei ministri, con la conseguenza che i dPCM trovano legittimazione nei decreti ex 77 Cost. e non già nella normativa della Protezione Civile.

Molteplici sono le tensioni a cui è sottoposta la Costituzione con l'adozione degli atti finora prodotti; in questo intervento si è deciso di valutare la tenuta delle limitazioni delle libertà via via definite e di cercare di proporre (con tutta la cautela del caso) un primo (parziale) bilancio 
sulla ragionevolezza delle misure adottate e, soprattutto, di valutare se queste si siano mosse nell'alveo del potere costituito.

Sempre a mo' di premessa metodologica, si sottolinea subito che fra le tante (tantissime) libertà costituzionali sottoposte a stress forse l'unica che al momento risulta formalmente indenne da precisi interventi è quella personale $^{32}$. Quindi, non entra nella nostra disamina l'art. 13 Cost., che tutela per l'appunto l'inviolabilità della libertà personale. Il che ha una notevole ripercussione sull'analisi da compiere e sulle risposte che si daranno, perché le garanzie poste alla libertà personale sono molto più stringenti rispetto alle altre, in quanto (ma lo stesso vale, ad esempio per il domicilio) il diritto è garantito non solo dall'istituto della riserva di legge (di tipo assoluto), ma altresì dalla riserva di giurisdizione.

Se - per come si è finora detto - la Costituzione italiana non conosce una precisa positivizzazione dello stato di emergenza, essa prevede però che le libertà possano essere limitate per motivi di sanità o di pubblica sicurezza o di incolumità pubblica, che si possa e si debba fare fronte ai «casi straordinari di necessità ed urgenza» (art. 77 Cost.) e che si possa deliberare lo stato di guerra (art. 78 Cost.). Che la situazione odierna rientri solo nel primo caso è in medias res ${ }^{33}$, e alla luce di ciò bisogna giudicare se è da considerarsi come legittima o meno l'attuale limitazione dei diritti. E infatti, se la Costituzione non prevede formalmente lo stato di emergenza (la tutela della salus rei publicae suprema lex esto) non significa d'altronde che non ci siano le misure costituzionalmente conformi per farvi fronte e che queste debbano avere precise caratteristiche, dovendo, id est, essere proporzionali, adeguate, ragionevoli, anche scientificamente ${ }^{34}$, e, soprattutto, adottate per un tempo limitato e circoscritto alla loro ineludibile necessità. La disciplina normativa che si analizzerà, quindi, non dovrebbe caratterizzarsi in termini 'assoluti' e 'rigidi', quanto piuttosto secondo un approccio metodologico mite. Mitezza che però - vale la pena sottolinearlo - deve significare flessibilità e non già indeterminatezza di regole che molte volte appaiono eccessivamente vaghe e prive di quella inequivocabilità che dovrebbe caratterizzarle per il rispetto della certezza del diritto ${ }^{35}$.

Fermo restando, ben inteso, che le limitazioni alle libertà sono possibili per motivi di sanità (o di sicurezza); tali limitazioni (data una 
formale catena normativa dell'emergenza) ${ }^{36}$ sono state introdotte - per garantire la riserva di legge (relativa) ${ }^{37}$, presente nell'intero articolato che presiede le libertà medesime - attraverso decreti-legge, con l'idea di offrire copertura normativa (abilitante) ${ }^{38}$ ai decreti del Presidente del Consiglio dei ministri (dPCM), delle Regioni, dei comuni e del Ministero della sanità (art. 32 l. n. 833/1978) per poter emanare ordinanze contingibili e urgenti in materia di igiene e sanità pubblica.

Le fonti del diritto emanate e adottate - anche per il contesto appena tratteggiato (di grande instabilità e di mutevolezza) ${ }^{39}$ - costituiscono una panoplia, un vero e proprio ginepraio, essendo al momento ben 57 gli atti del Governo, mentre è quasi fuori controllo ${ }^{40}$ il numero degli atti locali, regionali, della protezione civile e dei vari ministeri, in special modo quello della Salute.

Gli atti che più propriamente hanno una diretta ripercussione sul tema oggetto di analisi sono 5 decreti-legge (il secondo dei quali abroga il primo $)^{41}, 19 \mathrm{dPCM}^{42}$ e $10 \mathrm{DM}$ della salute ${ }^{43}$.

I diritti costituzionali che conoscono una limitazione sono quelli del lavoro (artt. 4 e 35 Cost.), di circolazione e di soggiorno (art. 16 Cost.), della libertà di riunione (art. 17), della libertà di culto (art. 19 Cost.), dell'istruzione (dell'infanzia, primaria inferiore e superiore, universitaria e di ulteriore specializzazione: artt. 33 e 34), delle attività economiche (art. 41 Cost.), del diritto di proprietà (art. 42), e delle stesse libertà politiche (rinvio delle elezioni amministrative e del referendum oppositivo ex art. 138 Cost.). Persino lo stesso diritto alla salute individuale (art. 32 Cost.) è stato limitato nella misura in cui tutte le cure 'non urgenti' sono state rinviate. Inoltre, molte delle previsioni - disposte interamente con decretolegge - attengono alla regolazione della giustizia e alla ripartizione delle competenze legislative e amministrative fra Stato e regioni.

In una situazione fisiologica è il Parlamento mediante lo strumento legislativo a poter limitare i diritti o a porli in bilanciamento tra di loro secondo ragionevolezza, dando seguito alla riserva di legge (assoluta e relativa) sempre presente per la tutela delle libertà. In situazione di emergenza, la disciplina è affrontata sì da una fonte primaria ma diversa dalla legge, vale a dire il decreto-legge (d.-l.) di cui all'art. 77 Cost. L'adozione dei decreti-legge è richiesta dall'eccezionalità del momento. 
La situazione di emergenza se, da una parte, permette e garantisce una rapida risposta ordinamentale attraverso l'adozione dei decretilegge, dall'altra, non dequota la Costituzione a mero orpello rimanendo nel tessuto ordinamentale tutte le garanzie di controllo dell'operato del Governo quando questi decide di predisporlo sotto la propria (collettiva) responsabilità. Tale fonte è sottoposta a un duplice controllo (oltre quelli che poi potranno attivarsi per la via giurisdizionale). Si tratta di controlli molto pervasivi perché o istantanei o da determinarsi in un lasso di tempo breve.

Il primo è esercitato dal Presidente della Repubblica che emana il d.-l. (e che può opporsi con un potere di veto) esercitando un controllo di tipo preventivo; il secondo, invece, rientra nel circuito della rappresentanza democratica, rimettendo al Parlamento la scelta sulla conversione o meno del contenuto del decreto; in caso di mancata conversione, gli effetti del decreto perdono efficacia in modo retroattivo. Il Parlamento, quindi, non viene spogliato dell'esercizio del potere legislativo e della sovranità popolare che rappresenta, e continua a esercitare una insostituibile fase di dibattito pubblico e trasparente tra la maggioranza e l'opposizione (che 'rimane agli atti'). Inoltre, il d.-l., in quanto fonte primaria, è sempre sindacabile dalla Corte costituzionale.

Fino a questo momento, però, la modalità di risposta all'emergenza ha dimostrato un'assoluta prevalenza del Governo e, al suo interno, del Presidente del Consiglio dei ministri. Come è facilmente verificabile con i dati più sopra riportati, a oggi il Parlamento ha occupato un ruolo decisamente marginale, almeno fino a quando è entrato in 'fase di conversione' in legge dei decreti-legge emanati. Inoltre, l'asse ParlamentoGoverno è stata nettamente sbilanciata a favore di quest'ultimo anche grazie a un uso 'parco' del decreto-legge e a un impiego massiccio dei $\mathrm{dPCM}$, come ordinanze extra ordinem ${ }^{44}$. Da questo punto di vista, l'impiego di una fonte anziché di un'altra ha delle notevoli conseguenze sulla tenuta della tutela delle libertà.

Se l'impiego del decreto-legge risponde alla necessità di limitare i diritti con una fonte del diritto primaria sottoposta a garanzie, l'utilizzo dei dPCM pone una serie di questioni, in quanto, a differenza dei d.-1. ${ }^{45}$, questi non sono atti con forza di legge, non sono regolamenti in senso 
proprio (e infatti non sono emanati dal Presidente della Repubblica e sono provvisori) e dovrebbero (sic!) essere impiegati per lo più per lo svolgimento di funzioni di c.d. 'alta amministrazione'.

I dPCM in parola, invece, contribuiscono a definire (in modo forte ovvero in modo debole) i limiti alle libertà. I dPCM pur se pensati per avere una funzione di attuazione e non di mera esecuzione, nel complesso non sembra che (in questa situazione di emergenza) pongano problemi di costituzionalità insuperabili, nella misura in cui il principio di legalità è garantito (nella sua veste sia formale che sostanziale ${ }^{46}$ ). Molte volte però la portata delle misure, seppure limitata da una elencazione di quelle da eseguire, pare nella possibilità di un potere elevatamente discrezionale, più di quello che normalmente pertiene al potere amministrativo (anche quando la riserva di legge è di tipo assoluto).

Qui bastino solo due esempi ${ }^{47}$ in cui il $\mathrm{dPCM}$ ha inciso sulle libertà (o sul principio di non discriminazione) in modo maggiore rispetto a quanto già fatto con il decreto-legge a cui doveva dare per l'appunto attuazione: se il d.-l. n. 6 del 23 febbraio configurava il divieto di allontanamento dal comune, è con il dPCM dell'8 marzo, prima, e del 9 marzo, poi, che si è inciso maggiormente sull'art. 16 Cost. nella misura in cui si è vietato lo spostamento delle persone all'interno dei territori comunali della Regione Lombardia, e poi dell'intero territorio nazionale. Se il d.-l. n. 19 del 25 marzo disponeva la limitazione della circolazione delle persone, anche prevedendo limitazioni alla possibilità di allontanarsi dalla propria residenza, domicilio o dimora - se non per spostamenti individuali limitati nel tempo e nello spazio o motivati da esigenze lavorative, da situazioni di necessità o urgenza (non meglio specificate e quindi affetti da indeterminatezza), da motivi di salute o da altre specifiche ragioni -, è solo con il dPCM del 26 aprile che si genera una diseguaglianza nella misura in cui si valutano come 'di necessità' gli spostamenti per incontrare congiunti e non già amici o persone fra di loro legate da rapporti sentimentali più 0 meno stabili; in quest'ultima previsione pare non rispettato il principio di legalità sostanziale.

Inoltre, l'abuso in quantità e qualità del $\mathrm{dPCM}$ mina alla stessa configurazione costituzionale del Presidente del Consiglio dei ministri ${ }^{48}$ che non è più (solo) colui che «dirige» la politica generale 
del Governo e «mantiene» l'unità di indirizzo politico e amministrativo promovendo e coordinando l'attività del Governo (come vorrebbe l'art. 95 della Costituzione), ma diventa organo individuale capace di adottare decisioni di tipo deontico.

La fase più critica è stata di certo quella verificatasi all'indomani della deliberazione dello stato di necessità, allorquando la situazione emergenziale obbligava la tutela del diritto alla salute come interesse della collettività in modo pieno e 'assoluto'.

La compressione delle libertà, per la tutela del (solo) diritto alla salute (collettiva) predisposta attraverso i dPCM non avrebbe dovuto trovare quello spazio ampio che invece le è stato riconosciuto, in quanto anche con l'adozione di decreti-legge (che producono immediatamente efficacia giuridica) poi (nel caso) attuati con dPR (atti governativi classicamente attuativi di disposizioni normative generali, predisposti collegialmente ed emanati e, quindi, controllati dal Presidente della Repubblica) si sarebbero potute garantire le stesse finalità, ma con la garanzia di maggiori controlli esterni. Quindi, almeno per il prossimo futuro (ma a guardare il modus operandi conseguente alle due proroghe dello stato di emergenza ciò appare più una petitio principii ${ }^{49}$, sarebbe auspicabile un maggiore utilizzo dello strumento della decretazione (come fonte di produzione e non sulla produzione), a maggior ragione da quando le misure di contenimento del virus hanno dimostrato che (anche) le Camere possono lavorare nel rispetto delle norme di sicurezza sanitaria ${ }^{50}$, dovendo esse riunirsi per svolgere un 'servizio pubblico essenziale'.

Assunto il passaggio effettuato per affrontare l'emergenza, dalle misure incentrate sul sistema della protezione civile a quelle sull'emergenza sanitaria e, infine, a quelle sul binomio d.-l-/dPCM ${ }^{51}$, quanto finora sostenuto - anche in termini critici - non ci permette però di giungere all'affermazione per cui tutti gli atti finora prodotti (per intenderci durante la c.d. 'fase $1{ }^{152}$ ) siano da assumersi come incostituzionali, in quanto la copertura formale e sostanziale assicurata col decreto-legge comunque c'è stata, anche se non sempre in maniera adeguata. Infatti, il primo d.-l. n. 6 del 2020 prevedeva all'art. 1 una serie di possibili limitazioni (seppure a maglie larghissime) dei diritti costituzionali, aggiungendo poi all'art. 2 una sorta di norma in bianco (questa in assoluto la disposizione più 
critica di tutte le norme emergenziali, perché non rispettosa della riserva di legge $)^{53}$, che (come più sopra già ricordato) riconosceva al Presidente del Consiglio dei ministri la possibilità di assumere tutte quelle «ulteriori misure» - adottate con dPCM - che fossero state ritenute necessarie, e non prevedeva alcuna temporaneità e nessun vincolo di proporzionalità delle misure indicate nei decreti del PCM. Tali atti potevano essere decretati, quindi, senza le dovute garanzie di controllo, seppure, ex art. 3, era comunque prevista, per la loro adozione, una previa acquisizione di pareri (non vincolanti) ovvero di concerto con alcuni Ministri e Presidenti di Regione interessati ${ }^{54}$.

Questa sorta di delega in bianco si poneva talmente al limite della tenuta costituzionale della normativa che si deve salutare con particolare favore (per il rispetto del principio stretto di legalità 'sostanziale') l'adozione (con una inversione dell'ordine cronologico delle fonti che è una caratteristica di tutta la fase emergenziale) del seguente e conseguente d.-l. n. 19, del 25 marzo 2020, che sostituisce la clausola di 'rinvio/delega in bianco' con una elencazione tassativa di tutte quelle misure che avrebbero potuto essere adottate sempre con lo strumento (flessibile) del dPCM.

Quindi, la normativa emergenziale finora prodotta è caratterizzata dal tandem decreto-legge e dPCM per la gestione complessiva dell'emergenza e per il bilanciamento e restrizione dei diritti e libertà fondamentali, e dall'adozione delle ordinanze (della Protezione civile ${ }^{55}$, dei singoli ministri e dei Presidenti di Regione e dei sindaci ${ }^{56}$ ) contingibili e urgenti per rispondere a questioni circoscritte e che richiedevano una velocità di risposta ancor più celere e maggiormente legata alla transitorietà della decisione amministrativa (attraverso atti provvisori).

Si riconosce, quindi, una 'patente di legittimità ${ }^{57}$ agli atti predisposti perché la 'fase 1' è stata caratterizzata da misure limitatrici che appaiono temporanee $^{58}$ (limitate alla fase emergenziale dichiarata e deliberata dal Consiglio dei ministri per il periodo dal 31 gennaio al 31 luglio 2020, ma con le delibere del 29 luglio 2020 e del 7 ottobre 2020 è stato prorogato lo stato di emergenza sul territorio nazionale relativo al rischio sanitario connesso all'insorgenza di patologie derivanti da agenti virali trasmissibili) ${ }^{59}$, adeguate, proporzionali e 'scientificamente' ragionevoli per la tutela della salute come interesse della collettività ${ }^{60}$. L'emergenza 
in questa fase è stata definita dalla modulazione dell'intensità delle limitazioni delle libertà con l'unico fine della tutela (il più possibile estesa) della salute.

\title{
UN BILANCIAMENTO OBBLIGATORIAMENTE VARIABILE NEL PASSAGGIO DA UNA FASE A UN'ALTRA DURANTE I TEMPI DEL COVID-19
}

\begin{abstract}
«Tutti i diritti fondamentali tutelati dalla Costituzione si trovano in rapporto di integrazione reciproca e non è possibile pertanto individuare uno di essi che abbia la prevalenza assoluta sugli altri. La tutela deve essere sempre 'sistemica e non frazionata in una serie di norme non coordinate e in potenziale conflitto tra loro'. Se così non fosse, si verificherebbe l'illimitata espansione di uno dei diritti, che diverrebbe 'tiranno' nei confronti delle altre situazioni giuridiche costituzionalmente riconosciute e protette, che costituiscono, nel loro insieme, espressione della dignità della persona» ${ }^{61}$.
\end{abstract}

Bisogna ora verificare se le misure finora adottate reggano a tale massima che è non solo dottrinaria, ma - per come si è appena visto anche giurisprudenziale. Se nella 'fase 1' dell'emergenza si è imposto il carattere preminente del diritto alla salute rispetto a tutti i diritti della persona, nella 'fase 2' - anche alla luce della tesi per cui una gerarchia tra diritti fondamentali non può darsi - è richiesto che il fisiologico, continuo e vicendevole bilanciamento tra princìpi e diritti fondamentali - senza pretese di «prevalenza assoluta di uno dei valori coinvolti, né il sacrificio totale di uno di loro» ${ }^{62}$ - sia rivalutato, e ciò è possibile solo attraverso una fonte primaria, e, quindi, data l'emergenza, attraverso il decreto-legge.

Il punto di equilibrio del bilanciamento potrà essere valutato in primis durante la conversione in Parlamento, nella consapevolezza che esso è dinamico e dovrà continuare a essere valutato secondo criteri di temporaneità, proporzionalità 63 e ragionevolezza, requisiti questi la cui presenza è necessaria perché non si sacrifichi il diritto nel suo nucleo essenziale.

Nella 'fase 1 ' i limiti sono stati determinati ex art. 16 Cost. in riferimento alla libertà di circolazione ${ }^{64}$ ("per motivi di sanità o di sicurezza») ed ex art. 17 Cost. in riferimento alla libertà di riunione («per comprovati motivi 
di sicurezza o di incolumità pubblica») e giudicati legittimi per la tutela dell'art. 32 Cost. A valle di queste limitazioni, tutte le altre libertà - più sopra richiamate - hanno trovato una contrazione 'costituzionalmente orientata' per rendere effettivi i limiti e quindi la tutela del bene salute, con la precisazione, però, che i limiti non sono mai stati di tipo assoluto (con sospensione del diritto) riconoscendo a tutti i diritti coinvolti la capacità della loro riespansione ('sono consentiti solo gli spostamenti motivati da comprovate esigenze lavorative o situazioni di necessità ovvero per motivi di salute'). Si afferma ciò, nella consapevolezza che la libertà di circolazione è prodromica a tutti gli altri diritti; limitata questa (\#iorestoacasa) si impedisce l'esercizio delle altre libertà. La 'fase 1' è stata caratterizzata dalla elevata «entità del pericolo» che ha determinato un concreto bilanciamento a favore del preminente interesse collettivo della salute. La ragionevolezza come parametro applicato in concreto (superata la 'fase 1') ha spinto a riconsiderare il bilanciamento in una fase in cui l'entità del pericolo è meno forte ${ }^{65}$ e richiede l'espansione della tutela di più diritti, la più ampia e progressiva possibile.

Anche se non è superata la fase pandemica, permanendo lo stato di emergenza sanitaria nazionale, l'odierna contingenza permette-(rebbe) di essere 'affrontata' con il solo strumento del decreto-legge/legge di conversione e con la produzione di decreti meramente esecutivi e non più attuativi (sempre se non si dovesse 'ritornare' nella c.d. 'fase 1') ${ }^{66}$.

La 'fase $2^{\prime 67}$, quindi, deve aprirsi alla necessità del ritorno agli ordinari rapporti tra poteri e alle competenze costituzionali. Questo dovrebbe essere possibile perché la 'necessità' di rispondere in modo celere a situazioni che non si conoscono e che mettono a serio repentaglio il bene vita è nettamente diminuita.

Lo strumento del decreto-legge, in questa fase, definita di transizione ${ }^{68}$, garantisce il pieno rispetto della riserva di legge, oltre al fatto di essere predisposto da un organo collegiale e di garantire una ben precisa catena dei meccanismi di controllo: del Presidente della Repubblica in sede di emanazione, del Parlamento in sede di conversione, nuovamente del Presidente della Repubblica in fase di promulgazione, e in ultimo della Corte costituzionale. 
Le norme devono essere disciplinate con fonte primaria e devono riguardare categorie nel loro complesso unitarie (in modo da non creare diseguaglianze: si pensi al riavvicinamento ai congiunti e non agli amici, all'apertura solo di alcuni e non anche di altri esercizi commerciali, a misure che potrebbero riguardare solo gli anziani e non già tutti i soggetti a rischio per co-morbilità, ...) e i limiti devono essere razionalmente adeguati alla fase dell'epidemia, quella nella quale 'si convive con il virus', nella misura in cui si riesce a limitarne al massimo la trasmissibilità.

Un (nuovo) decreto-legge, insomma, che definisca una (nuova) cornice ogni qual volta ciò sia reso necessario dalla concreta evoluzione degli studi epidemiologici, a esempio nel passaggio da una fase a un'altra. Lo strumento unilaterale del dPCM nell'attuale contingenza (che ha trovato una apertura sostanzialmente 'scaglionata' e una, seppur tardiva e non 'adeguata' attivazione dell'applicazione c.d. 'Immuni'69) non è per sua natura in grado di definire il bilanciamento dei diritti; e ciò non solo da un punto di vista formale ma anche sostanziale, in quanto il grado di discrezionalità da adottare sarebbe notevolmente ampio, non dovendosi, il $\mathrm{dPCM}$, limitare a 'scegliere' da un elenco già stilato quali misure adottare $\mathrm{e}^{70}$. Il quadro è ora diverso, dovuto a un bilanciamento (come dire?) 'più bilanciato'. Si dovrebbe procedere non più per sottrazione, ma per decisione valutativa di tipo proattivo.

E infatti, è stato emanato il d.l. n. 33 del 16 maggio, anche se esso continua a richiamare per la sua attuazione non tanto i dPR, quanto piuttosto i dPCM.

Con l'art. 1 del d.l. n. 33 del 16 maggio 2020 Ulteriori misure urgenti per fronteggiare l'emergenza epidemiologica da CoViD-19, il quadro è totalmente cambiato: «cessano di avere effetto tutte le misure limitative della circolazione all'interno del territorio regionale di cui agli articoli 2 e 3 del decreto-legge 25 marzo 2020, n. 19, e tali misure possono essere adottate o reiterate, ai sensi degli stessi articoli 2 e 3 , solo con riferimento a specifiche aree del territorio medesimo interessate da particolare aggravamento della situazione epidemiologica». Il nuovo bilanciamento ${ }^{71}$ (che richiede - avrebbe richiesto - anche una nuova fase di collaborazione tra Enti vieppiù leale) fa sì che da una fase di limitazione generalizzata delle libertà si sia passati ad una in cui è possibile fare tutto ciò che non è 
espressamente vietato ${ }^{72}$. Anche se non a tutti i diritti è stata riconosciuta (fin da subito) una più ampia espansione, il che desta più di una perplessità.

Se le libertà ora sono limitate nella giusta misura in cui si riescono a garantire le c.d. 'misure di protezione con distanziamento sociale (fisico)' tanto che si sono riaperte tutte le attività commerciali, ampliato il diritto di riunione in luogo pubblico, privato e aperto al pubblico (cinema, palestre e teatri), resa possibile la partecipazione alle funzioni religiose - il diritto all'istruzione (anche universitaria) ha continuato a essere 'regolato' come se si fosse ancora in 'fase 1' e non è stato permesso il rientro alle lezioni in presenza ${ }^{73}$ per un tempo molto più lungo (fino all'inizio del nuovo anno scolastico e/o accademico). Al netto di una 'precauzione del contagio' non è stata motivata la scelta per cui si può andare in vacanza, in piscina, in palestra, ... (sempre con il limite del divieto di assembramento ex art.1, c. 8; e comunque seguendo il dato dei dati epidemiologici) e non anche a scuola o all'Università. Detto in altro modo, ciò che è mancata è la previsione per cui le misure limitative al diritto in parola 'possano essere adottate nel rispetto dei principi di adeguatezza e di proporzionalità'.

Dicevamo che un ruolo importante continua ad essere assolto dal dPCM e non pare risolutiva (al fine di un controllo preventivo di tipo efficace) la sua 'parlamentarizzazione'. In sede di conversione del d.l. n. 19 è stato aggiunto un periodo all'art. 2, c. 1, per il quale il Presidente del Consiglio dei ministri o un Ministro da lui delegato «illustra preventivamente alle Camere il contenuto dei provvedimenti da adottare ai sensi del presente comma, al fine di tenere conto degli eventuali indirizzi dalle stesse formulati; ove ciò non sia possibile, per ragioni di urgenza connesse alla natura delle misure da adottare, riferisce alle Camere». Quindi, il parere non è vincolante e l'urgenza, che caratterizza in re ipsa lo stato di emergenza, potrebbe rendere nulla la previsione normativa appena richiamata ${ }^{74}$.

L'espressione «tenere conto» è una formula vieppiù blanda rispetto a quella a cui idealmente rinvia (art. 7, c. 1, l. n. 234/2012), ovverosia quella per cui il Governo, preso atto dell'indirizzo definito da parte dell'organo parlamentare, «assicura che la posizione rappresentata dall'Italia in sede di Consiglio dell'Unione europea ovvero di altre istituzioni od organi 
dell'Unione sia coerente con gli indirizzi definiti dalle Camere in relazione all'oggetto di tale posizione».

Anche il mero riferimento all'obbligo di riferire alle Camere le misure adottate non prevede (per obbligo) - come ovvio che sia - alcun voto camerale. Quindi, di fatto, il dPCM non viene 'parlamentarizzato'.

\section{LA NECESSARIETÀ DELLA REDUCTIO AD UNUM CON GIUSTIFICAZIONE DELLE ECCEZIONI, PER UNA CONDUZIONE UNITARIA DELL'EMERGENZA, ALMENO NELLA 'FASE 1'. NELLA 'FASE 2' ... PROVE DI DIFFERENZIAZIONE (MOTIVATE) IN MODALITÀ STOP AND GO}

Trattare della ragionevolezza dei limiti ai diritti fondamentali richiede, altresì, la consapevolezza che su di essi possono incidere, con una moltitudine di atti (non solo legislativi) una pluralità di Enti, fin qui ampiamente richiamati, in primis le Regioni e i Comuni.

Affrontare una emergenza pandemica ha una sua capacità (complessivamente) reattiva, adeguata nella misura in cui almeno la direzione sia affidata a un vertice le cui determinazioni non possano essere messe in discussione appena dopo essere state adottate ${ }^{75}$, con la conseguenza che è la situazione di emergenza alla quale vuole darsi una soluzione a non trovare una risposta adeguata e neanche risolutiva.

Come si è ricordato poche pagine più sopra, un contemperamento delle esigenze della differenziazione (attraverso la chiamata in sussidiarietà) ${ }^{76}$ è richiesto per il tempo straordinario dello stato di necessità.

Un protagonismo decentrato, in una certa misura anche permesso da una disciplina permissiva, è stato riconosciuto da entrambi i primi decreti-legge emanati.

Il d.-l. n. 6, poi abrogato dal n. 19, normava la disciplina nel suo art. 3, c. 2: «[n]elle more dell'adozione dei decreti del Presidente del Consiglio dei ministri di cui al comma 1 , nei casi di estrema necessità e urgenza le misure di cui agli articoli 1 e 2 possono essere adottate ai sensi dell'articolo 32 della legge 23 dicembre 1978, n. 833, dell'articolo 
117 del decreto legislativo 31 marzo 1998, n. 112, e dell'articolo 50 del testo unico delle leggi sull'ordinamento degli enti locali, approvato con decreto legislativo 18 agosto 2000, n. 267».

I poteri emergenziali, come si evince dalla normativa richiamata dallo stesso d.-l., erano già nella disposizione dei Presidenti di Regione e dei sindaci, quindi, al fine di dare una precedenza omogeneizzante ai dPCM attuativi del d.-l. si limitava il potere emergenziale alla presenza cumulativa di due condizioni, vale a dire «nelle more» dei dPCM e nei «casi di estrema necessità ed urgenza». Il problema interpretativo, che non favoriva la reductio ad unum, era quello per cui era lasciata alla discrezione (politico-istituzionale) degli organi di vertice degli esecutivi la definizione di quali fossero le concrete misure da adottare oltre l'interpretazione per cui l'espressione «nelle more» poteva comportare che il dies a quo ripartiva all'indomani dell'adozione di un nuovo dPCM. Il profluvio degli atti adottati (una vera e propria ipertrofia) dimostra la inapplicabilità dell'articolo appena richiamato ${ }^{77}$.

Un obiettivo da raggiungere in tempi brevi è stato quello di limitare le spinte localistiche con l'introduzione di vincoli ancora più rigorosi. Il d.-l. n. 19 ha quindi prescritto ex art. 2, c. 2, che

«[n]elle more dell'adozione dei decreti del Presidente del Consiglio dei ministri ${ }^{78}$ di cui al comma 1 e con efficacia limitata fino a tale momento, in casi di estrema necessità e urgenza per situazioni sopravvenute le misure di cui all'articolo 1 possono essere adottate dal Ministro della salute ai sensi dell'articolo 32 della legge 23 dicembre 1978, n. 833».

Le ordinanze, quindi, secondo tale lettura potevano essere adottate dal solo Ministro della salute e non più dai Presidenti di regione e dai sindaci. Mentre a questi ultimi era (nei fatti) 'inibito' l'esercizio del potere - in modo diretto ${ }^{79}$ ma anche indiretto ${ }^{80}$, rimanendo uno spazio di intervento residuale - alle Regioni continuava a essere riconosciuto il potere d'ordinanza ${ }^{81}$ limitatamente all'introduzione di misure restrittive che - seppur «nelle more» ${ }^{82}$ - potevano essere adottate «esclusivamente nell'ambito delle attività di loro competenza e senza incisione delle attività produttive e di quelle di rilevanza strategica per l'economia nazionale». 
Le autonomie regionali e gli enti locali durante la fase emergenziale devono, quindi, garantire l'adeguatezza (delle misure) e la differenziazione (delle valutazioni) al fine di rispondere a livello locale alle esigenze di emergenza nazionale in modo sempre più istantaneo ed efficiente. E in effetti:

«in presenza di emergenze di carattere nazionale, dunque, pur nel rispetto delle autonomie costituzionalmente tutelate, vi deve essere una gestione unitaria della crisi per evitare che interventi regionali o locali possano vanificare la strategia complessiva di gestione dell'emergenza, soprattutto in casi in cui non si tratta solo di erogare aiuti o effettuare interventi ma anche di limitare le libertà costituzionali» 83 .

Il cambio di paradigma è poi avvenuto con il decreto n. 33. La prima fase dell'emergenza ha richiesto una riconduzione a unità per una uniformità delle analisi epidemiologiche per relative misure. Queste vanno mantenute, ma allo stesso tempo una gestione maggiormente aperta alle autonomie e quindi alla differenziazione è richiesta anche per rispondere alle concrete situazioni epidemiologiche su base locale.

Quindi da una capacità di differenziazione solo in peius è stato poi possibile esercitare il potere di autonomia anche in melius (almeno per le attività economiche, produttive e sociali).

L'esercizio della differenziazione è possibile secondo il noto criterio di cedevolezza:

Le attività economiche, produttive e sociali devono svolgersi nel rispetto dei contenuti di protocolli o linee guida idonei a prevenire o ridurre il rischio di contagio nel settore di riferimento o in ambiti analoghi, adottati dalle regioni o dalla Conferenza delle regioni e delle province autonome nel rispetto dei principi contenuti nei protocolli o nelle linee guida nazionali. In assenza di quelli regionali trovano applicazione i protocolli o le linee guida adottati a livello nazionale. Le misure limitative delle attività economiche, produttive e sociali possono essere adottate, nel rispetto dei principi di adeguatezza $e$ proporzionalità, con provvedimenti emanati ai sensi dell'articolo 2 del decreto-legge n. 19 del 2020 o del comma 16.

E quindi, secondo l'art. 1, c. 16, d.-l. n. 33, è permissibile un sistema che ha in sé una forma notevole di elasticità nel rispetto di vincoli di natura sia sostanziale che procedurale: 
Per garantire lo svolgimento in condizioni di sicurezza delle attività economiche, produttive e sociali, le regioni monitorano con cadenza giornaliera l'andamento della situazione epidemiologica nei propri territori e, in relazione a tale andamento, le condizioni di adeguatezza del sistema sanitario regionale. I dati del monitoraggio sono comunicati giornalmente dalle regioni al Ministero della salute, all'Istituto superiore di sanità e al comitato tecnico-scientifico di cui all'ordinanza del Capo del dipartimento della protezione civile del 3 febbraio 2020, n. 630, e successive modificazioni. In relazione all'andamento della situazione epidemiologica sul territorio, accertato secondo i criteri stabiliti con decreto del Ministro della salute del 30 aprile 2020 e sue eventuali modificazioni, nelle more dell'adozione dei decreti del Presidente del Consiglio dei ministri di cui all'articolo 2 del decreto-legge $n$. 19 del 2020, la Regione, informando contestualmente il Ministro della salute, può introdurre misure derogatorie, ampliative o restrittive, rispetto a quelle disposte ai sensi del medesimo articolo 2.

I provvedimenti regionali, quindi, richiedono motivazioni e basi scientifiche su cui basare le proprie misure di differenziazione, e ciò attraverso una vera e propria fase istruttoria.

Ma la curva epidemiologica (che rileva un processo) ha ancora una volta imposto la ridefinizione delle relazioni tra Enti limitando e vincolando la capacità decisionale delle Regioni che, in una fase (quella odierna, in cui la diffusione del virus, col quale si deve continuare a convivere, è ritornata particolarmente estesa e contagiosa) che richiede il ritorno ad una gestione centrale e centralizzata delle misure su cui le Regioni possono eventualmente intervenire ma solo con restrizioni più stringenti; le eventuali misure ampliative non possono più essere assunte mediante una mera comunicazione, ma solo previa intesa con il Ministro della salute. La modifica (con d.-l. n. 125/2020) è la seguente:

«restrittive rispetto a quelle disposte ai sensi del medesimo articolo 2, ovvero, nei soli casi e nel rispetto dei criteri previsti dai citati decreti e d'intesa con il Ministro della salute, anche ampliative».

Ritorna ancora la elasticità delle relazioni che sul principio della leale collaborazione dovrebbero assicurare la regia allo Stato che ha la competenza esclusiva nella determinazione dei livelli essenziali dei diritti e in riferimento alla profilassi internazionale, fra le altre materie. 
Stante l'evolversi della situazione epidemiologica, così come il carattere particolarmente diffusivo dell'epidemia e l'incremento dei casi sul territorio nazionale, benché si sia ancora in 'fase 2' si assiste ad una continua messa a punto del concreto bilanciamento tra diritti con prevalenza della tutela della salute e restrizioni della socialità (libertà di riunione), rimodulazioni della modalità di erogazione della didattica (incremento delle forme a distanza per gli ultimi anni degli istituti scolastici di secondo grado) e decongestionamento dei servizi di trasporto pubblico. Si procede, quindi, con gradualità, al fine di controllare l'andamento del quadro epidemiologico con l'intento di evitare il ritorno alla 'fase 1' (lockdown nazionale). Tenendo conto dell'evoluzione del quadro pandemico territoriale, fra le misure introdotte con dPCM del 18 ottobre, si prevedono modalità chiusure territorialmente specifiche anche se definite con poca chiarezza circa l'organo competente ad adottare le decisioni in parola:

"[d]elle strade o piazze nei centri urbani, dove si possono creare situazioni di assembramento, può essere disposta ${ }^{84}$ la chiusura al pubblico, dopo le ore 21,00 , fatta salva la possibilità di accesso, e deflusso, agli esercizi commerciali legittimamente aperti e alle abitazioni private».

\section{STATI EMERGENZIALI, SALVAGUARDIA DELLA SICUREZZA E DIRITTI FONDAMENTALI NEGLI ORDINAMENTI DI DEMOCRAZIA PLURALISTA. CONCLUSIONI}

Ritornando al tema principale di questa riflessione deve rilevarsi come gli ordinamenti contemporanei (e quello italiano fra essi) evidenzino ormai una notevole tensione nella ricerca degli equilibri e dei bilanciamenti fra i richiamati beni giuridici parimenti fondamentali (la protezione della libertà personale nelle sue diverse manifestazioni costituzionali e quella della sicurezza). Il diritto alla sicurezza, in tale prospettiva, viene colto sempre meno nell'ottica della teorica hobbesiana di fondamento del potere, valorizzandosene, piuttosto, quelle garanzie a suo tempo 
positivizzate nell'art. 2 della Déclaration des droits de l'home et du citoyen, che la collocavano fra i diritti (giusnaturalistici e) inalienabili dell'uomo ${ }^{85}$, secondo il quale "[l] e but de toute association politique est la conservation des droits naturels et imprescriptibles de l'homme. Ces droits sont la liberté, la propriété, la sûreté, et la résistance à l'oppression».

A fronte delle trasformazioni dei contesti nazionali e internazionali - determinate dai processi di globalizzazione, dai tentativi di controllo delle risorse del pianeta nonché dei flussi migratori (in modo particolare dei richiedenti asilo) - e a fronte della emersione di minacce terroristiche, prima, e ora epidemiologiche (vieppiù diffuse e aggressive), la sicurezza (intesa come 'esigenza di sicurezza', come situazione giuridica 'pretensiva' di protezione, di cura da parte della 'Repubblica') si connota al contempo come garanzia di continuità dello Stato e delle relative funzioni di protezione della pace sociale e come bene giuridico legato in modo inscindibile alla persona, al bene della vita, alla sua dignità. Accanto al tradizionale inquadramento e alle relative garanzie costituzionali delle (diverse manifestazioni della) libertà personale come potere (mandato allo Stato) volto ad assicurare il superamento del bellum omnium contra omnes, perseguendo in tal modo l'obiettivo della pace sociale, nella evoluzione più recente del costituzionalismo contemporaneo, così, ritroviamo nuove garanzie fra cui - oltre a un ricco catalogo di diritti fondamentali - il diritto alla sicurezza, inteso come bene giuridico autonomo (come 'sicurezza di'), come diritto fondamentale della persona, oggetto, in sede di giurisdizione costituzionale, di significativi bilanciamenti con le garanzie accordate alle libertà personali.

In tale ottica, la sicurezza, in quanto diritto della persona, deve «procedere insieme con le libertà dell'individuo ed essere così concepita come un obiettivo cui deve tendere l'azione dello Stato e degli altri pubblici poteri, sempre nel pieno rispetto del nucleo essenziale degli altri diritti garantiti dalla Costituzione» ${ }^{86}$. In altri termini, la sicurezza, inscritta nel progetto costituzionale definito dalle previsioni costituzionali in tema di eguaglianza sostanziale fra i soggetti ritrova la sua realizzazione nell'attuazione dei principi e dei diritti fondamentali della Costituzione attraverso l'azione del legislatore e degli altri soggetti territoriali costitutivi della Repubblica e attraverso le garanzie della giurisdizione ordinaria e 
costituzionale per farne valere le disposizioni contro lo stesso legislatore (eventualmente) 'ingiusto'. In una simile prospettiva, così, la sicurezza si concretizza nella piena attuazione delle previsioni dello Stato di diritto e delle finalità dello Stato costituzionale sociale, positivizzando in tal modo uno stretto rapporto fra concezione della democrazia, forma di Stato e diritti fondamentali. E infatti, avendo a mente ciò che l'esperienza della emergenza sanitaria sta provocando in Italia, possiamo affermare che la Costituzione si mantiene ${ }^{87}$.

La dichiarazione dello stato di emergenza nazionale è una procedura prevista per legge e la Costituzione permette al decreto-legge di rispondere prontamente alle situazioni emergenziali e di essere allo stesso tempo controllato da organi terzi. Non si è dinanzi a nessuna lacuna costituzionale; l'eventuale previsione in Costituzione di una disposizione circa lo stato di emergenza dovrà in ogni caso rifuggire la possibilità di affidare a chiunque i 'pieni poteri', che non sono ipotizzabili neanche a seguito di dichiarazione di guerra ${ }^{88}$.

Ma se anche per il futuro qualcuno volesse intraprendere la strada della previsione di una clausola costituzionale dell'emergenza - pur nella oggettiva constatazione che «l'assenza di previsioni puntuali non significa mutismo costituzionale» ${ }^{89}$ - lo dovrà fare tenendo a mente le parole di Ackerman che, scrivendo a futura memoria, ammoniva ricordando che «[d]elineare un regime costituzionale per uno stato limitato di emergenza è una faccenda complicata. Se non si assumono attente precauzioni, le misure di emergenza hanno la tendenza a perpetuarsi ben oltre il tempo in cui sono necessarie» ${ }^{90}$.

La disciplina costituzionale dello stato d'emergenza dovrebbe avere il senso non già di attribuire poteri extra ordinem, che di fatto si affermerebbero in re ipsa con la necessità, quanto piuttosto delimitarne i confini di un potere che se libero nei fini potrebbe comportare una rottura. Detto in altri modi, la clausola di emergenza non dovrebbe che rispettare i fondamenti del costituzionalismo, quindi, del limite al potere. Così possono leggersi le clausole presenti negli altri ordinamenti costituzionali, ma anche nella stessa Convenzione dei diritti e delle libertà fondamentali, nel cui articolo 15, c. 1, si permette una deroga 'con limiti' 91 : 
"[i]n caso di guerra o in caso di altro pericolo pubblico che minacci la vita della nazione, ogni Alta Parte contraente può adottare delle misure in deroga agli obblighi previsti dalla presente Convenzione, nella stretta misura in cui la situazione lo richieda e a condizione che tali misure non siano in conflitto con gli altri obblighi derivanti dal diritto internazionale».

Intanto, durante la contingenza (che può essere sia di una situazione emergenziale che fisiologica) al legislatore dobbiamo richiedere un costante bilanciamento tra diritti, tale da limitare al massimo la contrazione di ognuno di essi e da non snaturare il corretto assetto dei poteri che deve essere sempre più retto dal principio di leale collaborazione. Senza mai pensare che le libertà possano essere sospese, perché ciò significherebbe mettere in discussione la stessa Costituzione che trova legittimazione proprio nella tutela dei diritti fondamentali ponendoci dinanzi a una contradictio in adiecto insostenibile.

In conclusione, quindi, si può affermare che l'Italia sta affrontando in modo proporzionale e adeguato l'emergenza sanitaria (al momento e comunque con tutte le criticità fin qui rilevate); una emergenza, questa, che a differenza di quella terroristica o di quelle naturali finora conosciute ha la particolarità di interessare contemporaneamente l'intero territorio nazionale, oltre che tutti i Paesi del mondo. Da tale prospettiva, una differenza nella capacità ordinamentale di risposta all'emergenza pandemica nella tutela delle libertà costituzionali si può rilevare non solo - come è ovvio che sia - tra i paesi democratici e quelli che non lo sono, ma anche fra quelli che rientrano nel medesimo regime di democrazia. Detto altrimenti, si registra una netta differenza fra le risposte all'emergenza pandemica alla luce della tenuta delle ragioni del costituzionalismo fra le democrazie mature e consolidate e quelle che anche autonomamente ${ }^{92}$ - si spingono a definirsi 'illiberali' (costituendo una degenerazione delle prime), in quanto solo queste ultime tendono ad 'usare' l'emergenza sanitaria per accentuare oltre l'indispensabile lo sbilanciamento dei poteri verso l'esecutivo (estendere sine die i poteri normativi del Governo e sostituire il controllo parlamentare con un generico obbligo di informazione $)^{93}$ e per forzare in modo evidentemente sproporzionato, inadeguato e indefinito i limiti alle libertà. 


\section{Alcune di queste esperienze dimostrano come la mera presenza di} una clausola costituzionalizzata dell'emergenza da sola non basti (anzi, tutt'altro) a porre limiti da considerarsi realmente tali (in quanto efficaci) alla destabilizzazione dei poteri a seguito dell'emergenza e, quindi, che il patologico non si trasformi in fisiologico.

\section{Note}

1 Da ultimi AA.VV., Stato di diritto. Emergenza. Tecnologia, a cura di M. Villone-G. De Minico, Consulta onLine, 2020.

2 B. Ackerman, La Costituzione di emergenza, Roma, 2005. Le citazioni nel testo sono di pp. 24 e 79.

3 U. Romagnoli, Art. 3, II comma, in G. Branca (cur.), Commentario della Costituzione, Roma, 1975.

4 Nella sent. cost. n. 275/2016, così, può leggersi che «è la garanzia dei diritti incomprimibili ad incidere sul bilancio, e non l'equilibrio di questo a condizionarne la doverosa erogazione».

5 G. Silvestri, Covid-19 e Costituzione, in UniCost.it, 04/10/2020; M. Luciani, Il sistema delle fonti del diritto alla prova dell'emergenza, in Liber amicorum per Pasquale Costanzo; G. Azzariti, Il diritto costituzionale d'eccezione, in www.costituzionalismo.it, 1/2020.

6 A. Di Giovine, La protezione della democrazia fra libertà e sicurezza, in Id., Democrazie protette e protezione della democrazia, Torino, 2005; P. Torretta, 'Diritto alla sicurezza' e (altri) diritti e libertà della persona: un complesso bilanciamento costituzionale, in A. D’Aloia (cur.), Diritti e Costituzione. Profili evolutivi e dimensioni inedite, Milano, 2003.

7 M. Dogliani, Il volto costituzionale della sicurezza, in Astrid Rassegna (27 aprile 2020).

8 G. Cerrina Feroni-G. Morbidelli, La sicurezza: un valore super-primario, in Percorsi costituzionali, $1 / 2008$, p. 33

9 L. Lorello, Il dilemma sicurezza vs. libertà al tempo del terrorismo internazionale, in AA.VV., Democracy and Security Review, 1/2017, p. 5.

10 G. Cerrina Feroni-G. Morbidelli, op.cit., p. 41.

11 A. Pace, La funzione di sicurezza nella legalità costituzionale, in Quaderni costituzionali, 4/2014.

12 A. Pace, op.cit.

13 L. Wurtz, Obsessions sécuritaires, in Le Monde diplomatique, richiamato da A. Di Giovine, op.cit.

14 M. Ruotolo, La sicurezza nel gioco del bilanciamento, in G. Cocco (cur.), I diversi volti della sicurezza, Milano, 2012, pp. 18 ss.

15 G. Peces-Barba, Teoria dei diritti fondamentali, Milano, 1993, pp. 223 ss.

16 T.F. Giupponi, Sicurezza personale, sicurezza collettiva e misure di prevenzione. La tutela dei diritti fondamentali e l'attività di intelligence, in Forum di Quaderni costituzionali, p. 3.

17 N. Bobbio, Sui diritti sociali, in G. Neppi Modona (a cura di), Cinquant'anni di Repubblica italiana, Torino, 1997.

18 G. De Vergottini, La difficile convivenza tra libertà e sicurezza: la risposta delle democrazie al terrorismo. Gli ordinamenti occidentali, in AA.VV., Annuario 2003. Libertà e sicurezza nelle democrazie contemporanee, Padova, 2003; T.E. Frosini, Il diritto costituzionale alla sicurezza, in www. forumdiquadernicostituzionali.it.

19 B. Ackerman, op.cit., p. 175.

20 B. Ackerman, op.cit., p. 24.

21 Ma quanto meno opportuna almeno da G. DE MINICO, Costituzionalizziamo l'emergenza?, in Osservatorio sulle fonti, fasc. speciale, 2020, pp. 542 ss.

22 Anche per questo la normativa presente nel Codice della protezione civile è stata ben presto superata, in quanto essa è stata pensata per altri tipi di calamità. Cfr., quindi, G. Di Cosimo, G. Menegus, L'emergenza Coronavirus tra Stato e Regioni: alla ricerca della leale collaborazione, in BioLaw Journal, special issue 1/2020, 185. 
23 Sulla particolarità della emergenza epidemiologica rispetto alle altre, cfr. A. Lauro, Urgenza e legalità ai tempi del Covid-19: fra limiti imprescindibili e necessaria flessibilità, in BioLaw Journal Instant Forum - Diritto, diritti ed emergenza ai tempi del Coronavirus, pp. 1 ss.

24 M. Cartabia, L'attività della Corte costituzionale nel 2019, in Cortecostituzionale.it, p. 18.

25 Per una precisazione non solo nominalistica, si rinvia infra a nota 66.

26 In un sistema, del resto, già gravato da vizi strutturali già di per sé allarmanti e che generano a loro volta diseguaglianza, inasprita dalla condizione di limitazione delle libertà personali: $\mathrm{S}$. Staiano, Né modello né sistema. La produzione del diritto al cospetto della pandemia, in Rivistaaic. it, 2/2020, p. 548; M. D’Amico, Emergenza, diritti, discriminazioni, in Gruppo di Pisa, 2/2020, p. 30 , in cui si parla di «discriminazione intersezionale».

27 Si avvertono le stesse preoccupazioni, quindi, di autorevole dottrina: G. Azzariti, I pieni e solitari poteri del capo del governo extra-ordinem, in "Il Manifesto", 19 marzo 2020. Cfr., altresì, F. Rescigno, La gestione del coronavirus e l'impianto costituzionale. Il fine non giustifica ogni mezzo, in Osservatorio Aic, 3/2020, 16 ss.

28 Con la successiva dichiarazione dell'Organizzazione mondiale della sanità dell'11 marzo 2020 l'epidemia da CoViD-19 è stata valutata come 'pandemia' in considerazione dei livelli di diffusività e gravità raggiunti a livello globale.

29 La durata dello stato di emergenza di rilievo nazionale non può superare i 12 mesi, ed è prorogabile per non più di ulteriori 12 mesi. In riferimento al crollo del Ponte Morandi, il d.-l. 30 dicembre 2019, n. 162, convertito con modificazioni dalla l. 28 febbraio 2020, n. 8, ha disposto può essere prorogato fino ad una durata complessiva di tre anni.

30 Tale d.lgs. costituisce la legislazione di emergenza nazionale (sulla costituzionalità di tale previsione si v., almeno, Corte cost., sent. 15/1982, 418/1992). I presupposti per la deliberazione (e rinnovo) stabiliti ex lege sono, ben inteso, sempre sindacabili. Si ricorda che non è previsto il controllo preventivo di legittimità della Corte dei conti.

31 Costituendo, questa seconda, una distinta «filiera» o «catena» differente seppur collegata alla prima (R. Romboli, L'incidenza della pandemia da Coronavirus nel sistema costituzionale italiano, in Consulta online, 3/2020, p. 519), in grado di approntare un quadro normativo speciale nel rispetto delle riserve di legge (M. Francaviglia, Decretazione d'urgenza, poteri di ordinanza e riserve di legge. La produzione normativa nell'emergenza Covid-19 alla luce del principio di legalità sostanziale, in Diritto pubblico, 2/2020, p. 371).

32 Conformemente a M. Bignami, Chiacchiericcio sulle libertà costituzionali al tempo del coronavirus, in Questione giustizia, p. 6; A. Cerri, A margine del coronavirus: note minime in tema di decreti del Presidente del Consiglio dei Ministri, in www.criticadeldiritto.it, p. 2: la restrizione alla libertà di circolazione è imposta direttamente dalla legge a una trasversale categoria di individui e non già dall'autorità amministrativa; venendo meno, quindi, il controllo dell'esercizio del potere amministrativo da parte dell'autorità giurisdizionale (riserva di giurisdizione) non rientriamo nella libertà personale $e x$ art. 13 Cost.; inoltre, rilevano non le colpe comportamentali di alcuno (che avrebbero la conseguenza della costrizione), ma sempre la tutela della salute collettiva e la limitazione della contagiosità del parassita. Per un'ampia disamina critica si v. R. Cherchi, A. Deffenu, Fonti e provvedimenti dell'emergenza sanitaria Covid-19: prime riflessioni, in www. dirittiregionali.it, $1 / 2020$, pp. 659 ss.

33 S. Staiano, op.cit., p. 532. Ci si permette di rinviare al nostro La metafora della guerra ai tempi del Coronavirus, in Corriere della Calabria, 1 aprile 2020. Contra, almeno A. Celotto, Necessitas non habet legem? Prime riflessioni sulla gestione costituzionale dell'emergenza coronavirus, Mucchi, Modena, 2020, 60.

34 A. Iannuzzi, Leggi "science driven" e CoVid-19. Il rapporto fra politica e scienza nello stato di emergenza sanitaria, in BioLaw Journal, special issue 1/2020, 119 ss. Fondamentale rimane lo studio di A. Spadaro, Sulle tre forme di "legittimazione" (scientifica, costituzionale e democratica) delle decisioni nello Stato costituzionale contemporaneo, in AA.VV., Bio-tecnologie e valori costituzionali. Il contributo della giurisprudenza costituzionale, a cura di A. D’Aloia, Torino, 2005, 580 ss.

35 M. Belletti, La "confusione" nel sistema delle fonti ai tempi della gestione dell'emergenza da Covid-19 mette a dura prova gerarchia e legalità, in Osservatorio costituzionale, 3/2020, 9, 12 ss.

36 M. Luciani, op.cit., p. 2. 
37 Corte cost., sent. n. 68/1964. Per la cui attuazione il rinvio è ai provvedimenti governativi nazionali, regionali e locali: F. Sorrentino, Riflessioni minime sull'emergenza Coronavirus, in Costituzionalismo.it, 1/2020, p. 136.

38 Una base legale attraverso una 'auto'-delega da parte dello stesso Governo. In dottrina almeno: A. Cardone, Il baratro della necessità e la chimera della costituzionalizzazione: una lettura della crisi delle fonti del sistema di protezione civile contro le battaglie di retroguardia, in Osservatorio sulle fonti, fasc. speciale, 2020, p. 327.

39 A. Morelli, Il Re del Piccolo Principe ai tempi del Coronavirus. Qualche riflessione su ordine istituzionale e principio di ragionevolezza nello stato di emergenza, in Diritti regionali, 1/2020, p. 519.

40 Calcolato con attenzione nella rubrica Emergenza Covid-19 curata da Osservaotriosullefonti.it.

41 Decreto-legge 23 febbraio 2020, n. 6, convertito, con modificazioni, dalla legge 5 marzo 2020, n. 13, e successivamente abrogato dal decreto-legge 25 marzo 2020, n. 19, convertito, con modificazioni, dalla legge 22 maggio 2020, n. 35. Decreto-legge 16 maggio 2020, n. 33, convertito, con modificazioni, dalla legge 14 luglio 2020, n. 74. Decreto-legge 30 luglio 2020, n. 83, convertito, con modificazioni, dalla legge 25 settembre 2020, n. 124. Decreto-legge 7 ottobre 2020, n. 125. Siamo in presenza di decreti 'autonomi' e non reiterati; sulla loro piena costituzionalità per tutti si rinvia a R. Romboli, op.cit., p. 522; M. Francaviglia, op. cit., pp. 369 s.

42 Il decreto-legge 23 febbraio 2020, n. 6, è stato attuato da 8 dPCM: del 23 febbraio; del 25 febbraio; del $1^{\circ}$ marzo; del 4 marzo; dell'8 marzo; del 9 marzo; dell'11 marzo; del 22 marzo.

Il decreto-legge 25 marzo 2020, n. 19, è tato attuato con 10 dPCM: del 1, del 10 e del 26 aprile; del 17 e del 18 maggio, dell'11 giugno, del 7 agosto 2020, del 7 settembre 2020 e del 13 ottobre 2020 poi modificato dal dPCM del 18 ottobre.

Il decreto-legge 16 maggio 2020, n. 33, è stato attuato con 8 dPCM: del 17 e del 18 maggio, dell'11 giugno, del 14 luglio, del 7 agosto 2020 , del 7 settembre 2020 e del 13 ottobre 2020 poi modificato dal dPCM del 18 ottobre.

43 Ordinanza del Ministro della salute 20 marzo 2020; ordinanza del Ministro della salute di concerto con il Ministro delle infrastrutture e dei trasporti 28 marzo 2020, con cui è stato disciplinato l'ingresso nel territorio nazionale tramite trasporto di linea aereo, marittimo, lacuale, ferroviario e terrestre. Decreto del Ministro della Salute 30 aprile 2020 Adozione dei criteri relativi alle attività di monitoraggio del rischio sanitario di cui all'allegato 10 del decreto del Presidente del Consiglio dei ministri del 26 aprile 2020. DMS della salute 9 e 16 luglio. Fra le più recenti anche i DDM della salute del 12 e del 16 agosto (nn. 202, 204), del 21 e del 25 settembre (nn. 234, 239), del 7 ottobre (n. 249).

44 E.C. Raffiotta, Sulla legittimità dei provvedimenti del Governo a contrasto dell'emergenza virale da Coronavirus, in Biolaw, 2/2020, p. 4.

45 Che comunque, durante la fase dell'emergenza, è come se si fossero tramutati da fonti di produzione a fonti sulla produzione: G. Mobilio, La decretazione d'urgenza alla prova delle vere emergenze. L'epidemia da Covid-19 e i rapporti tra decreto-legge e altre fonti, in Osservatorio sulle fonti, fasc. speciale, 2020, pp. 357 ss.

46 M. Francaviglia, op. cit., 376.

47 G. Di Cosimo, Tra decreti e decreti: l'importanza di usare lo strumento giusto, in LaCostituzione. info., pp. 1-3; G. Battistella, Stato di necessità e bilanciamenti costituzionali: sulla proporzionalità delle misure di contrasto al CoVid-19, in BioLaw Journal, special issue 1/2020, 157; M. Belletti, op.cit., 9.

48 Critico sull'"uomo solo al comando", M. Belletti, op.cit., 22; F. Rescigno, La gestione del coronavirus e l'impianto costituzionale. Il fine non giustifica ogni mezzo, in Osservatorio Aic, 3/2020, 15.

49 Il tutto ora scadenzato da una ri-accelerazione delle decisioni che si susseguono in un tempo strettissimo data la preoccupante situazione epidemiologica (ottobre 2020), che vede una progressione esponenziale dei numeri (aumentano in 'progressione geometrica') e che richiede di essere affrontata con rapidità per il contenimento del contagio assicurate dalla celerità tali da poter essere assunte con dPCM (sempre nel rispetto del d.-l. emanato). Si v., comunque, infra nota 74 .

50 Sul ruolo ricoperto dal Parlamento, per tutti, C. Tripodina, La Costituzione al tempo del Coronavirus, in Costituzionalismo.it, 1/2020, p. 85. 
51 Sui motivi che hanno indotto il governo a seguire la strada del d.-l. anziché delle sole ordinanze del Ministero della Salute e della Protezione civile, cfr., per tutti M. Cavino, Covid-19. Una prima lettura dei provvedimenti adottati dal Governo, in www.federalismi.it, 18 marzo 2020, p. 6; C. Pinelli, Il precario assetto delle fonti impiegate nell'emergenza sanitaria e gli squilibrati rapporti fra Stato e Regioni, in Rivista di Diritti comparati, 1/2020, p. 6: “[u]na delle ragioni può essere consistita nella percezione dell'inadeguatezza delle normative di settore dettate in riferimento a diversi tipi di emergenza (quella sulla protezione civile come quella sulla sanità) di fronte a un'epidemia che per aggressività e generalità di portata non era mai stata sperimentata in epoca repubblicana". In tema, altresì, A. Cardone, op.cit., p. 333; G. Mobilio, op.cit., p. 353.

52 Per 'fase 1 ' si intende quella in cui vige il pieno lockdown e, quindi, il divieto (sorretto da elevate sanzioni amministrative, penali nel d.-l. 6/2020 poi abrogato) imposto alle persone di accedere a un territorio, a un'area, di circolare liberamente, ..., fatti salvi comprovati (autocertificati) casi di necessità o di salute e che possono variare per intensità (zone rosse) in specifiche aree territoriali.

53 Tale art. 2 del d.-l- n. 6 (del 23 febbraio), consentendo la possibilità dell'adozione di ulteriori misure non ricomprese fra quelle previste a mo' di elenco, rappresentava una misura palesemente incostituzionale, tanto che è stata abrogata dal (non di poco) successivo d.-l. n. 19 (del 25 marzo).

54 Tale delega in bianco ha avuto una critica quasi unanime dalla dottrina costituzionalistica, qui ci si limita a citare G. Silvestri, op.cit., p. 3; M. Luciani, op.cit., p. 3; A. Lucarelli, Costituzione, fonti del diritto ed emergenza sanitaria, in Rivistaaic.it, 2/2020, p. 572. Per non rispettare, altresì, la tassatività e la determinatezza che la riserva di legge (sia assoluta che relativa) impone: F. Rescigno, La gestione del coronavirus e l'impianto costituzionale. Il fine non giustifica ogni mezzo, in Osservatorio Aic, 3/2020, 4.

55 Si v., quindi, la Delibera del Consiglio dei ministri del 31 gennaio 2020. Si ricorda che grazie al d.lgs. n. 1 del 2 gennaio 2018 Codice della Protezione civile, art. 25, si possono adottare le ordinanze (motivate e derogatorie della legislazione), e con esse, a esempio, è stato istituito il Comitato tecnico-scientifico che è organo nevralgico per l'adozione di tutti i dPCM (e non solo) e per garantire ragionevolezza scientifica alla decisione poi adottata dell'organo politico. Con tali ordinanze si è anche disposta la fornitura della strumentazione e degli ormai famosi dispositivi di protezione personale, in special modo dopo la nomina del Commissario Arcuri. Sui comitati di esperti si rinvia, per tutti, allo studio di A. Iannuzzi, Leggi "science driven", ecc., cit., 123 ss.

56 L'art. 35 d.-l. 2 marzo 2020, n. 19, vieta l'adozione di tali ordinanze: «[a] seguito dell'adozione delle misure statali di contenimento e gestione dell'emergenza epidemiologica da CoViD-19 non possono essere adottate e, ove adottate sono inefficaci, le ordinanze sindacali contingibili e urgenti dirette a fronteggiare l'emergenza predetta in contrasto con le misure statali», ma anche «regionali», aggiunta che si deve alla legge di conversione (l. n. 35/2020). La disciplina ora è nel d.-l. n. 33/2020, che ribadisce quanto appena riportato. Cfr. G. Alberico, Le ordinanze contingibili e urgenti nella gestione dell'emergenza sanitaria: il ruolo dei Sindaci nella disciplina del D.L. 33/2020, in Dirittifondamentali.it, 1 giugno 2020, 1 ss.; N. PignATELLI, La specialità delle ordinanze dei Sindaci nell'emergenza sanitaria nazionale: un potere "inesauribile", in Diritti regionali, 2/2020, 81. TAR Campania, Napoli sez. I, dec., 1 giugno 2020, n. 1120. Si v. infra nota 84.

57 Illegittima perché non rispondente ai criteri esposti la chiusura dei porti che viola il diritto alla salute e alla vita (oltre quello d'asilo) dei migranti. Nello stesso senso A. Algostino, Covid-19: primo tracciato per una riflessione nel nome della Costituzione, p. 4.

58 E. Grosso, Legalità ed effettività negli spazi e nei tempi del diritto costituzionale dell'emergenza. È proprio vero che "nulla potrà più essere come prima"?, in Federalismi, 16/2020, p. 6.

59 La proroga è possibile secondo l'art. 24 del medesimo articolato. È comunque previsto che siano sempre presenti i presupposti per la deliberazione dello stato di emergenza nazionale (art. 7, c. 1, lett. c)), vale a dire che si tratti di eventi che devono essere fronteggiati con mezzi e poteri straordinari, ovvero nella loro imminenza. Per come si cercherà di dimostrare nel corso della trattazione, la 'fase 2' della pandemia (si scrive nel mese di ottobre durante il quale è in corso la c.d. seconda ondata) è (e sarà) caratterizzata da un andamento variabile dal quale si può anche desumere che l'emergenza sanitaria (pur rimanendo tale) può (poteva, avrebbe potuto) essere fronteggiata con mezzi e poteri ordinari. Si afferma ciò perché una proroga non 
dovrebbe fondarsi sul mero timore (basato sul futuribile) e, quindi, sulla mera previsione di un aggravamento dell' evento calamitoso, richiedendo piuttosto essa che vi sia uno stato attuale di emergenza. Con il che non si vuole di certo affermare che in ragione di una mutata intensità o estensione dell'emergenza non si possa proclamare, e senza indugi, lo stato d'emergenza nazionale ex novo, entrando tale ipotesi nella responsabilità (sempre sindacabile) del Governo. Come sindacabile rimane la scelta della proroga. Assunto il modo di procedere del Governo italiano (si è prorogato il termine prima al 15 ottobre, poi al 31 gennaio) si può assumere che lo stato di emergenza sarà prorogato fino all'inizio della c.d. 'fase 3 ', vale a dire quella caratterizzata dal rilancio economico stante l'adozione di cure realmente efficaci o, molto più probabilmente, la validazione e la capillare distribuzione di uno o più vaccini.

Si sottolinea che mentre la dichiarazione dello stato di emergenza è stata deliberata dal Consiglio dei ministri conformemente a quanto disposto dal codice di protezione civile, le proroghe, invece, sono state subito seguite dalla predisposizione di un apposito decreto-legge in modo tale da permettere la discussione parlamentare in fase di conversione.

60 Si sta stressando questo carattere perché il diritto alla salute non va inteso nella sua dimensione liberale e individuale di rifiuto delle cure, la cui materia è rimessa interamente al consenso informato, ma alla dimensione collettiva della salute e del sistema sanitario nel suo complesso. Anche per ragioni di aziendalizzazione del sistema sanitario e di diffusione capillare (in specie nella parte settentrionale del Paese) di una politica sanitaria diretta verso una spinta privatizzazione della stessa con conseguente cambio di paradigma della cura vieppiù ospedalocentrica e quindi di cura e non già di prevenzione della patologia; oltre alla drastica riduzione di personale sanitario e infrastrutturale che ha messo letteralmente in ginocchio il sistema ospedaliero che sarebbe presto collassato se avesse conosciuto per un tempo prolungato un costante afflusso di pazienti. Da qui, l'obbligatorietà delle misure a favore della cura della salute individuale per la tutela di quella collettiva. Non si vuole tacere anche solo per denunciare ciò che fino a qui ha anche depauperato la ricerca scientifica.

61 Corte cost., sent. n. 85/2013, p.to 9 cons. dir.

62 Corte cost., sent. n. 58/2018, p.to 3.1. cons. dir.

63 Ai principi di adeguatezza e proporzionalità fa' ora espresso riferimento l'art. 1, c. 2, d.-l. n. 19/2020. È d'altronde sempre aperta la strada al ricorso giurisdizionale per sindacare i dPCM presuntivamente adottati in difformità a tali principi. Nella giurisprudenza della Corte costituzionale, il principio di proporzionalità è utilizzato come sinonimo di ragionevolezza o unitamente a esso o, comunque, mai come un vero e proprio test, così come autorevolmente ricordato da $\mathrm{M}$. Cartabia, I principi di ragionevolezza e di proporzionalità nella giurisprudenza italiana, Relazione predisposta per la Conferenza trilaterale della Corti costituzionali italiana, portoghese e spagnola, Roma, Palazzo della Consulta 24-26 ottobre 2013, nel sito telematico della Corte costituzionale, 6 . In altre esperienze di giustizia costituzionale, infatti (si v. 5), il principio di proporzionalità (così come teorizzato da R. Alexy, Teoría de los derechos fundamentales, Madrid, 2007, e come ricorda l'autorevole Autrice) si basa su una sequenza standardizzata degli atti del legislatore e suddivisa in quattro fasi: 1 . «di legittimità», vale a dire verifica della conformità ai principi fondamentali; 2 . «di valutazione delle connessione razionale», stante la valutazione che vi deve essere tra i mezzi (predisposti) e i fini (da perseguire); 3. «della verifica della necessità» del minor sacrificio che viene comunque richiesto ad altri diritti o beni costituzionalmente rilevanti a seguito della scelta legislativa compiuta; 4. «della proporzionalità in senso stretto», nel quale vengono esaminati gli effetti dell'atto legislativo nella concretezza dell'ordinamento (il bilanciamento).

64 G. Di Cosimo, Quel che resta della libertà di circolazione al tempo del Coronavirus, Osservatorio sulle fonti, fasc. speciale, 2020, pp. 566; R. Romboli, op.cit., p. 538.

65 In una fase in cui la tutela della salute (collettiva) trova fattualmente una (sempre maggiore) protezione: l'ormai famosa contagiosità R-t del virus è inferiore a R-1 e molto vicino a R-0.

66 Lo Stato può decidere le condizioni sostantive di rischio sanitario; del resto, lo scrive il dPCM del 26 aprile nel suo testo e nei suoi allegati che sono in chiusura riassunti nei diagrammi di flussi dell'allegato n. 10. In dottrina si rinvia almeno ad A. Lucarelli, op.cit., p. 566.

67 Durante la quale siamo chiamati a convivere con la presenza minacciosa del virus, disponendo ora di misure di prevenzione della diffusione del contagio (distanziamento anti droplet, mascherine, 
igienizzazione, ...), di un livello avanzato di conoscenza dello stato epidemiologico del contagio e di misure atte a contenerlo con strumenti diagnostici (tamponi e test sierologici). Tutta questa conoscenza era inimmaginabile e inimmaginata solo qualche mese fa. Ciò dato, cambia lo stato della contingenza e della valutazione della ragionevolezza del bilanciamento. Differentemente da quanto si ripete con insistenza - dal 3 giugno scorso (avvio degli spostamenti interregionali e di quasi tutte le attività private) - non si è quindi entrati nella c.d. 'fase 3', avendo appena definito la 'fase 2' come quella di 'convivenza con il virus'. Nel momento in cui si scrive - sono da poco stati superati le evidenze 'incoraggianti' degli studi epidemiologici presentati durante il periodo estivo - è quantomeno difficile definire con precisione quando inizierà e come sarà la 'fase 3', per il semplice motivo che, senza la commercializzazione di un vaccino, non è possibile prevedere la fine della 'fase 2'. La 'fase 3' dovrà essere molto diversa da quella che si è aperta lo scorso 3 giugno, perché si entrerà in tale fase quando si sancirà la fine dell'emergenza (dello stato di emergenza nazionale, pur sempre prorogabile) e il ripristino della massima tutela delle libertà e con essa della ricostruzione e del rilancio economico. Da tale momento in avanti non saranno più possibile deroghe legittimate da una emergenza che sarà finita.

68 G. Azzariti, Per la transizione decreti-legge e leggi ordinarie, in "Il Manifesto", 30 aprile 2020. Del resto, non si sta negando che un certo margine di esercizio di discrezionalità amministrativa sia per ciò solo da escludersi, perché esso è fisiologicamente sempre previsto.

69 Con la 'predisposizione' di un preciso bilanciamento fra i diritti della salute (e non solo) e della privacy: C. Bergonzini, Non solo privacy. Pandemia, contact tracing e diritti fondamentali, C. Colapietro, A. Iannuzzi, App di contact tracing e trattamento dei dati con algoritmi: la falsa alternativa fra tutela del diritto alla salute e protezione dei dati personali, entrambi in Dirittifondamentali.it, 2/2020, pp. 735 ss.

70 Tale elenco è stato da ultimo incrementato con l'art. 1, c. 1, lett. a) del d.-l. 125/2020 attraverso l'aggiunta della lettera hh-bis.

71 A.G. Lanzafame, Il lockdown, l'avvio della "fase due», e i problemi della "fase tre». La gestione dell'emergenza, sanitaria ed economica, da Covid-19 tra diseguaglianze ingiuste e diseguaglianze necessarie, in Dirittifondamentali.it, 2/2020, 498 ss.

72 0. Chessa, Il permesso di essere liberi, in LaCostituzione.info (22 maggio 2020); M. D’Amico, Op.cit., p. 28. Questa fase è comunque 'instabile' perché la pervasività dei limiti sarà mutevole dati il concreto andamento della curva epidemiologica e la capacità di gestione del territorio e della rete ospedaliera.

73 Per tali attività, quindi, ha continuato a trovare applicazione l'art. 2 del d.-l. n. 19 del 2020. Si v. nota precedente.

74 Così come avvenuto, del resto, per il dPCM del 18 ottobre 2020 che ha modificato il dPCM entrato in vigore solo qualche giorno prima (il 13 ottobre) e con esso le misure urgenti di contenimento del contagio sull'intero territorio nazionale.

75 Con gravi conseguenze sugli stessi studi epidemiologici con affievolimento della loro capacità predittiva.

76 Art. 1, c. 2, d.-l. 6/2020, ma anche del n. 19. Sono materie che rientrano nella potestà concorrente: protezione civile, salute, istruzione, valorizzazione dei beni culturali e ambientali. Tra quelle residuali: assistenza sociale, industria, commercio, artigianato, turismo, ecc.

77 Anche se una interpretazione restrittiva (e più che ragionevole) era assolutamente possibile: M. Luciani, op.cit., pp. 20 ss.; R. Cherchi, A. Deffenu, op.cit., p. 672.

78 Da adottarsi anche su proposta del Ministro della Salute, del Presidente della Regione (rispetto alle competenze) e anche previo parere, non vincolante, di altri ministri e dai Presidenti delle Regioni interessate (se lo sono tutte, dal Presidente della Conferenza delle Regioni). Sulle conferenze, almeno: E. Catelani, Centralità della Conferenza delle Regioni e delle Province autonome durante l'emergenza Covid-19? Più forma che sostanza, in Osservatorio sulle fonti, n. 2/2020, pp. $502 \mathrm{ss}$.

79 Art. 3, c. 2, d.-l. n. 19/2020: «[i] Sindaci non possono adottare, a pena di inefficacia, ordinanze contingibili e urgenti dirette a fronteggiare l'emergenza in contrasto con le misure statali, né eccedendo i limiti di oggetto cui al comma 1». 
80 Nella misura in cui misure vieppiù restrittive sono ora ad appannaggio delle Regioni e tale possibilità è formalmente espressa, il contrasto si ha (al di là della concreta portata della misura se più o meno liberale non rileva) ogni qualvolta si disciplina in modo differente la stessa materia. Ai sindaci, quindi, permane il potere emergenziale limitatamente a ciò che non trova disciplina (statale e regionale) alcuna, limitatamente alla partizione territoriale sulla quale si ha competenza.

81 Art. 3, c. 1, d.-l. n. 19/2020.

82 Di un dPCM di cui non si sa neanche la eventuale adozione, con la conseguenza che il dPCM non ha un termine massimo di efficacia.

83 Così Cons. St., sez. I, parere del 7 aprile 2020, n. 735. P.to 8.5 Considerato.

84 La ratio della disposizione in parola è quella di evitare un lockdown generalizzato, attraverso misure di chiusura temporalmente e territorialmente circoscritte. Competente a predisporre tali eventuali misure sarà - oltre il sindaco che dispone del potere di ordinanza contingibile ed urgente ex artt. 50 e 54 TUEL -, per sua natura, il Comitato provinciale per l'ordine e la sicurezza pubblica, un collegio di organi presieduto dal prefetto e composto dal questore, dal sindaco del comune capoluogo di provincia, dal presidente della Provincia, dai comandanti provinciali dei Carabinieri e della Guardia di finanza e dai sindaci degli altri comuni eventualmente interessati. Il compito di dar seguito alle future ordinanze emesse dai sindaci, per i conseguenti controlli, dovrà essere affidato alla polizia di Stato, ai carabinieri e alla polizia municipale.

85 T.E. Frosini, op.cit., 2.

86 Ibid., p. 2.

87 A. Ruggeri, Il coronavirus, la sofferta tenuta dell'assetto istituzionale e la crisi palese, ormai endemica, del sistema delle fonti, in Consulta onLine, 1/2020, p. 204; U. Allegretti, E. Balboni, Autonomismo e centralismo nella lotta contro la pandemia coronavirus, in Forum di quaderni costituzionali, 1/2020, p. 543; S. Staiano, op.cit., p. 532; U. De Siervo, Emergenza covid e sistema delle fonti: prime impressioni, in Osservatorio sulle fonti, fasc. speciale, 2020, p. 309.. Molto interessante, anche da questo punto di vista TAR Calabria (Sezione Prima), sent. 9 maggio 2020, n. 841. 'Anticipata' da U. Adamo, S. Gambino, W. Nocito, La Regione Calabria, il DPCM del 26 aprile e le "Ordinanze Santelli" nn. 37 e 38 alla luce di una non trovata leale collaborazione, in Astrid Rassegna, 7/2020, pp. 1 ss., e annotata da F. Pagano, A. Saitta, F. Saitta, Il giudice amministrativo stoppa la ripartenza anticipata della Regione Calabria: sul lockdown è lo Stato a dettare legge, in OsservatorioAic.it, 3/2020, pp. 1 ss.; A. Saporito, Il principio di leale collaborazione al tempo dell'emergenza sanitaria, in Dirittifondamentali.it, 2/2020, pp. 887 ss.; In tema, cfr. E. Longo, Episodi e momenti del conflitto Stato-regioni nella gestione della epidemia da Covid-19, in Osservatorio sulle fonti, fasc. speciale, 2020 , pp. 398 ss.

88 In tal caso i poteri sarebbero comunque 'necessari': G. Silvestri, op.cit., p. 1.

89 M. Luciani, op.cit., p. 25.

90 B. Ackerman, op.cit., p. 15

91 Sottoposti a loro volta ad uno stretto controllo di proporzionalità: R. Lugarà, Emergenza sanitaria e art. 15 CEDU: perché la Corte europea dovrebbe intensificare il sindacato sulle deroghe ai diritti fondamentali, in Osservatorio costituzionale AIC, 3/2020, pp. 341 ss.

92 Non potendosi pensare - neanche semanticamente - ad una democrazia che sia illiberale: A. Spadaro, Dalla "democrazia costituzionale" alla "democrazia illiberale" (populismo sovranista), fino alla.... "democratura", in www.dpceonline.it, p. 3878.

93 Come in Ungheria: legge organica n. 12 del 2020; ampiamente in tema J. Sawicki, La pandemia Covid-19, in Polonia e in Ungheria, come possibile occasione per intensificare la mutazione illiberale 2/delle istituzioni, in DPCE online, 2/2020, pp. 1963 ss. Stessa analisi anche per il caso polacco (J. Sawicki, op.cit., p. 1967) in cui si assiste ad una torsione illiberale di istituti di protezione anche non proclamando lo stato di emergenza ma solo allo scopo di non attivare il rinvio automatico delle elezioni presidenziali 


\section{REFERÊNCIAS}

AA.VV., Stato di diritto. Emergenza. Tecnologia, a cura di M. Villone-G. De Minico, Consulta onLine, 2020.

B. Ackerman, La Costituzione di emergenza, Roma, 2005. Le citazioni nel testo sono di pp. 24 e 79.

U. Adamo, S. Gambino, W. Nocito, La Regione Calabria, il DPCM del 26 aprile e le "Ordinanze Santelli" nn. 37 e 38 alla luce di una non trovata leale collaborazione, in Astrid Rassegna, $7 / 2020$.

G. Alberico, Le ordinanze contingibili e urgenti nella gestione dell'emergenza sanitaria: il ruolo dei Sindaci nella disciplina del D.L.33/2020, in Dirittifondamentali. it, 1 giugno 2020.

R. Alexy, Teoría de los derechos fundamentales, Madrid, 2007.

A. Algostino, Covid-19: primo tracciato per una riflessione nel nome della Costituzione.

U. Allegretti, E. Balboni, Autonomismo e centralismo nella lotta contro la pandemia coronavirus, in Forum di quaderni costituzionali, 1/2020.

G. Azzariti, I pieni e solitari poteri del capo del governo extra-ordinem, in "Il Manifesto", 19 marzo 2020.

G. Azzariti, Per la transizione decreti-legge e leggi ordinarie, in "Il Manifesto", 30 aprile 2020 .

G. Battistella, Stato di necessità e bilanciamenti costituzionali: sulla proporzionalità delle misure di contrasto al CoVid-19, in BioLaw Journal, special issue 1/2020.

M. Belletti, La "confusione" nel sistema delle fonti ai tempi della gestione dell'emergenza da Covid-19 mette a dura prova gerarchia e legalità, in Osservatorio costituzionale, $3 / 2020$.

C. Bergonzini, Non solo privacy. Pandemia, contact tracing e diritti fondamentali, Dirittifondamentali.it, 2/2020. 
M. Bignami, Chiacchiericcio sulle libertà costituzionali al tempo del coronavirus, in Questione giustizia.

N. Bobbio, Sui diritti sociali, in G. Neppi Modona (a cura di), Cinquant'anni di Repubblica italiana, Torino, 1997.

A. Cardone, Il baratro della necessità e la chimera della costituzionalizzazione: una lettura della crisi delle fonti del sistema di protezione civile contro le battaglie di retroguardia, in Osservatorio sulle fonti, fasc. speciale, 2020.

M. Cartabia, I principi di ragionevolezza e di proporzionalità nella giurisprudenza italiana, Relazione predisposta per la Conferenza trilaterale della Corti costituzionali italiana, portoghese e spagnola, Roma, Palazzo della Consulta 24-26 ottobre 2013, nel sito telematico della Corte costituzionale.

M. Cartabia, L'attività della Corte costituzionale nel 2019, in Cortecostituzionale.it.

E. Catelani, Centralità della Conferenza delle Regioni e delle Province autonome durante l'emergenza Covid-19? Più forma che sostanza, in Osservatorio sulle fonti, n. $2 / 2020$.

M. Cavino, Covid-19. Una prima lettura dei provvedimenti adottati dal Governo, in www.federalismi.it, 18 marzo 2020.

A. Celotto, Necessitas non habet legem? Prime riflessioni sulla gestione costituzionale dell'emergenza coronavirus, Mucchi, Modena, 2020.

A. Cerri, A margine del coronavirus: note minime in tema di decreti del Presidente del Consiglio dei Ministri, in www.criticadeldiritto.it.

G. Cerrina Feroni-G. Morbidelli, La sicurezza: un valore super-primario, in Percorsi costituzionali, $1 / 2008$.

R. Cherchi, A. Deffenu, Fonti e provvedimenti dell'emergenza sanitaria Covid-19: prime riflessioni, in www.dirittiregionali.it, 1/2020.

O. Chessa, Il permesso di essere liberi, in LaCostituzione.info (22 maggio 2020). 
C. Colapietro, A. Iannuzzi, App di contact tracing e trattamento dei dati con algoritmi: la falsa alternativa fra tutela del diritto alla salute e protezione dei dati personali, Dirittifondamentali.it, 2/2020.

M. D’Amico, Emergenza, diritti, discriminazioni, in Gruppo di Pisa, 2/2020.

G. De Minico, Costituzionalizziamo l'emergenza?, in Osservatorio sulle fonti, fasc. speciale, 2020 .

U. De Siervo, Emergenza covid e sistema delle fonti: prime impressioni, in Osservatorio sulle fonti, fasc. speciale, 2020.

G. De Vergottini, La difficile convivenza tra libertà e sicurezza: la risposta delle democrazie al terrorismo. Gli ordinamenti occidentali, in AA.VV., Annuario 2003. Libertà e sicurezza nelle democrazie contemporanee, Padova, 2003.

G. Di Cosimo, Quel che resta della libertà di circolazione al tempo del Coronavirus, Osservatorio sulle fonti, fasc. speciale, 2020.

G. Di Cosimo, Tra decreti e decreti: l'importanza di usare lo strumento giusto, in LaCostituzione.info.

G. Di Cosimo, G. Menegus, L'emergenza Coronavirus tra Stato e Regioni: alla ricerca della leale collaborazione, in BioLaw Journal, special issue 1/2020.

A. Di Giovine, La protezione della democrazia fra libertà e sicurezza, in Id., Democrazie protette e protezione della democrazia, Torino, 2005.

M. Dogliani, Il volto costituzionale della sicurezza, in Astrid Rassegna (27 aprile 2020).

M. Francaviglia, Decretazione d'urgenza, poteri di ordinanza e riserve di legge. La produzione normativa nell'emergenza Covid-19 alla luce del principio di legalità sostanziale, in Diritto pubblico, 2/2020.

T.E. Frosini, Il diritto costituzionale alla sicurezza, in www. forumdiquadernicostituzionali.it. 
T.F. Giupponi, Sicurezza personale, sicurezza collettiva e misure di prevenzione. La tutela dei diritti fondamentali e l'attività di intelligence, in Forum di Quaderni costituzionali.

E. Grosso, Legalità ed effettività negli spazi e nei tempi del diritto costituzionale dell'emergenza. È proprio vero che "nulla potrà più essere come prima"?, in Federalismi, 16/2020.

A. Iannuzzi, Leggi "science driven" e CoVid-19. Il rapporto fra politica e scienza nello stato di emergenza sanitaria, in BioLaw Journal, special issue 1/2020.

A.G. Lanzafame, Il lockdown, l'avvio della "fase due», e i problemi della "fase tre». La gestione dell'emergenza, sanitaria ed economica, da Covid-19 tra diseguaglianze ingiuste e diseguaglianze necessarie, in Dirittifondamentali.it, 2/2020.

A. Lauro, Urgenza e legalità ai tempi del Covid-19: fra limiti imprescindibili e necessaria flessibilità, in BioLaw Journal Instant Forum - Diritto, diritti ed emergenza ai tempi del Coronavirus.

E. Longo, Episodi e momenti del conflitto Stato-regioni nella gestione della epidemia da Covid-19, in Osservatorio sulle fonti, fasc. speciale, 2020.

L. Lorello, Il dilemma sicurezza vs. libertà al tempo del terrorismo internazionale, in AA.VV., Democracy and Security Review, 1/2017.

R. Lugarà, Emergenza sanitaria e art. 15 CEDU: perché la Corte europea dovrebbe intensificare il sindacato sulle deroghe ai diritti fondamentali, in Osservatorio costituzionale AIC, 3/2020.

G. Mobilio, La decretazione d'urgenza alla prova delle vere emergenze. L'epidemia da Covid-19 e i rapporti tra decreto-legge e altre fonti, in Osservatorio sulle fonti, fasc. speciale, 2020.

A. Morelli, Il Re del Piccolo Principe ai tempi del Coronavirus. Qualche riflessione su ordine istituzionale e principio di ragionevolezza nello stato di emergenza, in Diritti regionali, $1 / 2020$.

A. Pace, La funzione di sicurezza nella legalità costituzionale, in Quaderni costituzionali, 4/2014. 
F. Pagano, A. Saitta, F. Saitta, Il giudice amministrativo stoppa la ripartenza anticipata della Regione Calabria: sul lockdown è lo Stato a dettare legge, in OsservatorioAic.it, 3/2020.

G. Peces-Barba, Teoria dei diritti fondamentali, Milano, 1993.

N. Pignatelli, La specialità delle ordinanze dei Sindaci nell'emergenza sanitaria nazionale: un potere "inesauribile", in Diritti regionali, 2/2020.

C. Pinelli, Il precario assetto delle fonti impiegate nell'emergenza sanitaria e gli squilibrati rapporti fra Stato e Regioni, in Rivista di Diritti comparati, 1/2020.

E.C. Raffiotta, Sulla legittimità dei provvedimenti del Governo a contrasto dell'emergenza virale da Coronavirus, in Biolaw, 2/2020.

F. Rescigno, La gestione del coronavirus e l'impianto costituzionale. Il fine non giustifica ogni mezzo, in Osservatorio Aic, 3/2020.

U. Romagnoli, Art. 3, II comma, in G. Branca (cur.), Commentario della Costituzione, Roma, 1975.

R. Romboli, L'incidenza della pandemia da Coronavirus nel sistema costituzionale italiano, in Consulta online, 3/2020.

A. Ruggeri, Il coronavirus, la sofferta tenuta dell'assetto istituzionale e la crisi palese, ormai endemica, del sistema delle fonti, in Consulta onLine, 1/2020.

M. Ruotolo, La sicurezza nel gioco del bilanciamento, in G. Cocco (cur.), I diversi volti della sicurezza, Milano, 2012.

A. Saporito, Il principio di leale collaborazione al tempo dell'emergenza sanitaria, in Dirittifondamentali.it, 2/2020.

J. Sawicki, La pandemia Covid-19, in Polonia e in Ungheria, come possibile occasione per intensificare la mutazione illiberale 2/delle istituzioni, in DPCE online, 2/2020.

G. Silvestri, Covid-19 e Costituzione, in UniCost.it, 04/10/2020; M. Luciani, Il sistema delle fonti del diritto alla prova dell'emergenza, in Liber amicorum per 
Pasquale Costanzo; G. Azzariti, Il diritto costituzionale d'eccezione, in www. costituzionalismo.it, $1 / 2020$.

F. Sorrentino, Riflessioni minime sull'emergenza Coronavirus, in Costituzionalismo. it, $1 / 2020$.

A. Spadaro, Dalla "democrazia costituzionale" alla "democrazia illiberale" (populismo sovranista), fino alla... "democratura", in www.dpceonline.it.

A. Spadaro, Sulle tre forme di "legittimazione" (scientifica, costituzionale e democratica) delle decisioni nello Stato costituzionale contemporaneo, in AA.VV., Bio-tecnologie e valori costituzionali. Il contributo della giurisprudenza costituzionale, a cura di A. D’Aloia, Torino, 2005.

S. Staiano, Né modello né sistema. La produzione del diritto al cospetto della pandemia, in Rivistaaic.it, 2/2020.

P. Torretta, 'Diritto alla sicurezza' e (altri) diritti e libertà della persona: un complesso bilanciamento costituzionale, in A. D'Aloia (cur.), Diritti e Costituzione. Profili evolutivi e dimensioni inedite, Milano, 2003.

C. Tripodina, La Costituzione al tempo del Coronavirus, in Costituzionalismo.it, $1 / 2020$.

Recebido em: 30/11/2020

Autor convidado

\section{Ugo Adamo}

Ricercatore di Istituzioni di Diritto Pubblico, Università della Calabria. ugo.adamo@unical.it. Ricercatore (RTD-B) di Istituzioni di diritto pubblico, Università della Calabria

\section{Silvio Gambino}

Professore Emerito, Università della Calabria. E-mail: silvio.gambino@unical.it

Università della Calabria, Dipartimento di Economia, Statistica e Finanza Giovanni Amania , Ponte Pietro Bucci, Cubo 1/C, II píano, 87036 Arcavacata di Rende. 
